\title{
Click chemistry approach to a series of calcitriol analogues with heterocyclic side chains
}

\author{
Zoila Gándara ${ }^{1}$, Pedro-Lois Suárez ${ }^{1}$, Alioune Fall ${ }^{2}$, Massène Sène ${ }^{2}$, Ousmane Diouf ${ }^{2}$, \\ Mohamed Gaye ${ }^{2}$, Generosa Gómez ${ }^{1, *}$ and Yagamare Fall ${ }^{1, *}$ \\ ${ }^{1}$ Departamento de Química Orgánica, Facultad de Química and Instituto de Investigación Biomedica (IBI), \\ University of Vigo, Campus Lagoas de Marcosende, 36310 Vigo, Spain \\ ${ }^{2}$ Laboratoire de Chimie de Coordination Organique (LCCO), Département de Chimie, Faculté des Sciences et \\ Techniques: Université Cheikh Anta Diop de Dakar, Sénégal
}

\begin{abstract}
We report a straightforward synthesis of a series of novel calcitriol analogues from vitamin $\mathrm{D}_{2}$ with some modification of the procedures described by Calverley and Choudhry. This approach allows the large scale synthesis of a late-stage intermediate common to all the analogues of the series.This intermediate was successfully employed to synthesize a huge number of calcitriol analogues using a "click" chemistry approach.
\end{abstract}

Keywords: Calcitriol; Vitamin $\mathrm{D}_{2}$; triazole; azaanalogue; "Click” chemistry.

\section{Introduction}

1,25-Dihydroxyvitamin $\mathrm{D}_{3}$ (1, calcitriol) (Fig.1), the hormonally active metabolite of vitamin $\mathrm{D}_{3}(2)$, acts as a regulator in calcium and phosphate homeostasis $^{1}$. Next to these classical activities, calcitriol has been shown to inhibit cellular proliferation and to induce cellular differentiation ${ }^{2}$. However the therapeutic utility of $\mathbf{1}$ is hampered by the effective doses leading to calcemic side effects and this has stimulated the search for analogues having a relatively weak systemic effect on calcium metabolism while maintainig potent regulatory effects on cell differentiation and proliferation.
As part of our ongoing program on the synthesis of vitamin D analogues modified at the side chain, ${ }^{3}$ we envisaged the synthesis of various calcitriol analogues having heteroatoms on their side chain. The rational that could explain this choice was: we have already synthesized Aza-vitamin D analogues ${ }^{3 \mathrm{r}}$ and the biological activity of some of these derivatives was later studied showing that they had less calcemic effect than calcitriol. The strategy we used so far involved construction of the triene unit on the CD fragment following the introduction of the side chain. This strategy is inconvenient if a lengthy series of analogues with modified side chains are to be prepared for systematic biological evaluation.<smiles>C=C1/C(=C\C=C2/CCC[C@]3(C)[C@@H]2CC[C@H]3[C@H](C)CCCC(C)(C)O)C[C@@H](O)C[C@H]1O</smiles><smiles>C=C1CC[C@H](O)C/C1=C/C=C1\CCC[C@]2(C)[C@H]1CC[C@@H]2[C@H](C)CCCC(C)C</smiles>

Figure 1. Structures of 1,25-Dihydroxyvitamin $D_{3}(\mathbf{1})$ and vitamin $D_{3}(2)$.

\section{Results and Discussion}

We examined the possibility of preparing a series of analogues modified at the side chain from a common intermediate, in which the labile triene system was already present. The use of this strategy involved the concept of the triene system protection to allow chemical modification of the vitamin D side chain. This concept received relatively little attention. ${ }^{4}$ Among these approaches, the one using the preparation and subsequent thermolysis of the sulfur dioxide adducts of vitamin $\mathrm{D}_{2}{ }^{4 \mathrm{~b}, \mathrm{c}}$ seemed to us more appropriate for a large scale synthesis of a latestage intermediate such as $\mathbf{1 0}$ (Scheme 1). 

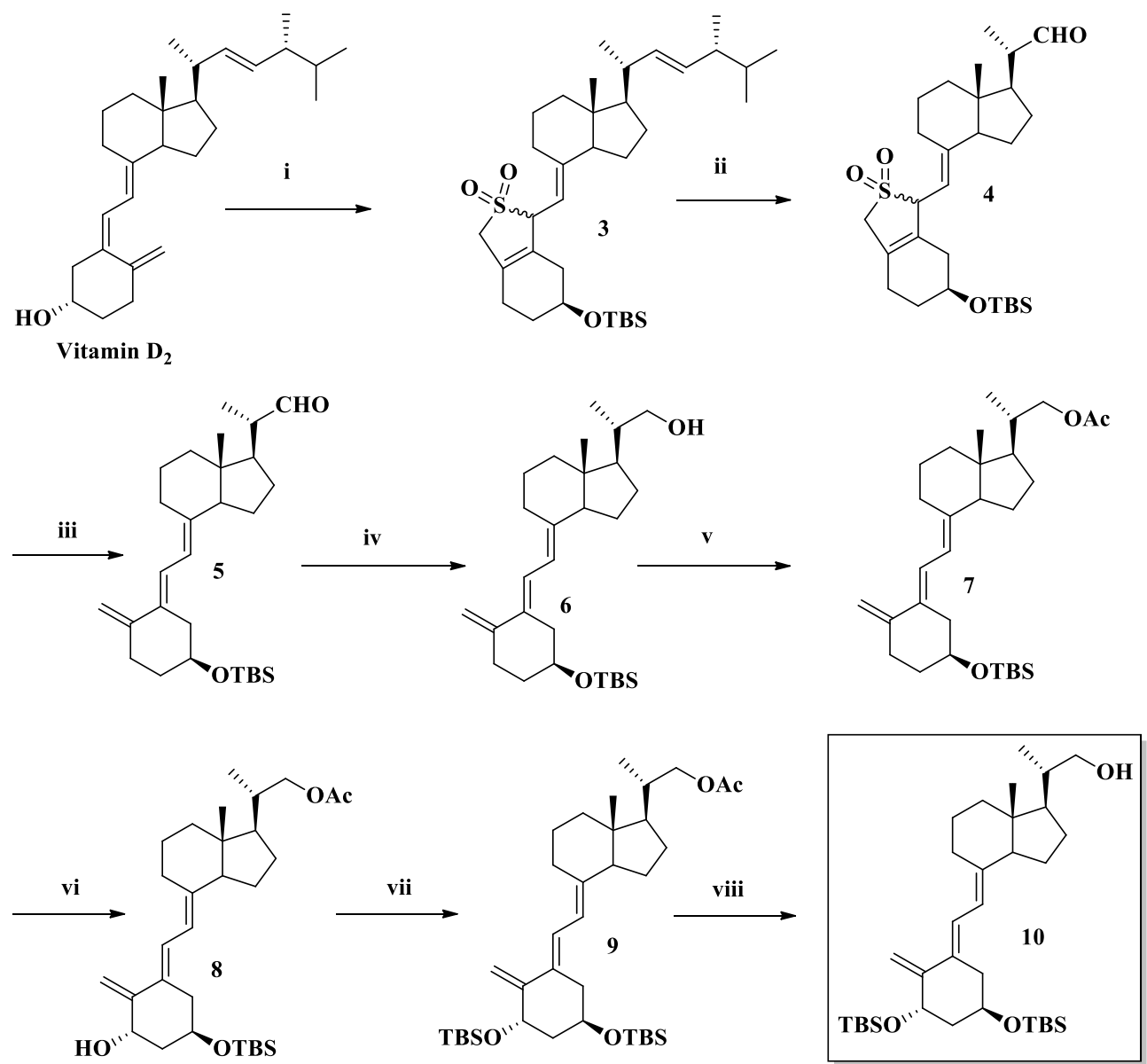

Scheme 1.Synthesis of intermediate 10 from vitamin $\mathrm{D}_{2}$. Reagents and conditions: (i) a) $\mathrm{SO}_{2}, \mathrm{CH}_{2} \mathrm{Cl}_{2},-25^{\circ} \mathrm{C}$ to $-10^{\circ} \mathrm{C}$; b) TBSCl, cat. DMAP, $\mathrm{CH}_{2} \mathrm{Cl}_{2},-5^{\circ} \mathrm{C}$ to rt $\left(97 \%\right.$, 2 steps); (ii) a) $\mathrm{O}_{3}, \mathrm{CH}_{2} \mathrm{Cl}_{2}, \mathrm{MeOH},-78^{\circ} \mathrm{C}$; b) $\mathrm{PPh}_{3}$,

$0^{\circ} \mathrm{C}$ to $25^{\circ} \mathrm{C}$; (iii) $\mathrm{NaHCO}_{3}, \mathrm{EtOH}$, reflux; (iv) $\mathrm{NaBH}_{4}, \mathrm{MeOH}, 0^{\circ} \mathrm{C}\left(85 \%\right.$ from 3); (v) $\mathrm{Ac}_{2} \mathrm{O}, \mathrm{Et}_{3} \mathrm{~N}, \mathrm{DMAP}$ (96\%); (vi) a) $\mathrm{SeO}_{2}, \mathrm{MeOH}$, reflux; b) 7, $\mathrm{NMO}, 50^{\circ} \mathrm{C}$ (60\%); (vii) TBSCl, imidazole, DMAP, $\mathrm{CH}_{2} \mathrm{Cl}_{2}$ (88\%); (viii) $\mathrm{K}_{2} \mathrm{CO}_{3}, \mathrm{MeOH}(94 \%)$.

Accordingly vitamin $\mathrm{D}_{2}$ was converted to its $\mathrm{SO}_{2}$-adducts 3 in $97 \%$ yield by dissolving in liquid sulfur dioxide and subsequent silylation. The ozonolysis of $\mathbf{3}$ resulted to be extremely troublesome and after much experimentation the best reaction conditions could be established. The results are summarized in Table 1.

Table 1. Ozonolysis of $\mathbf{3}$.
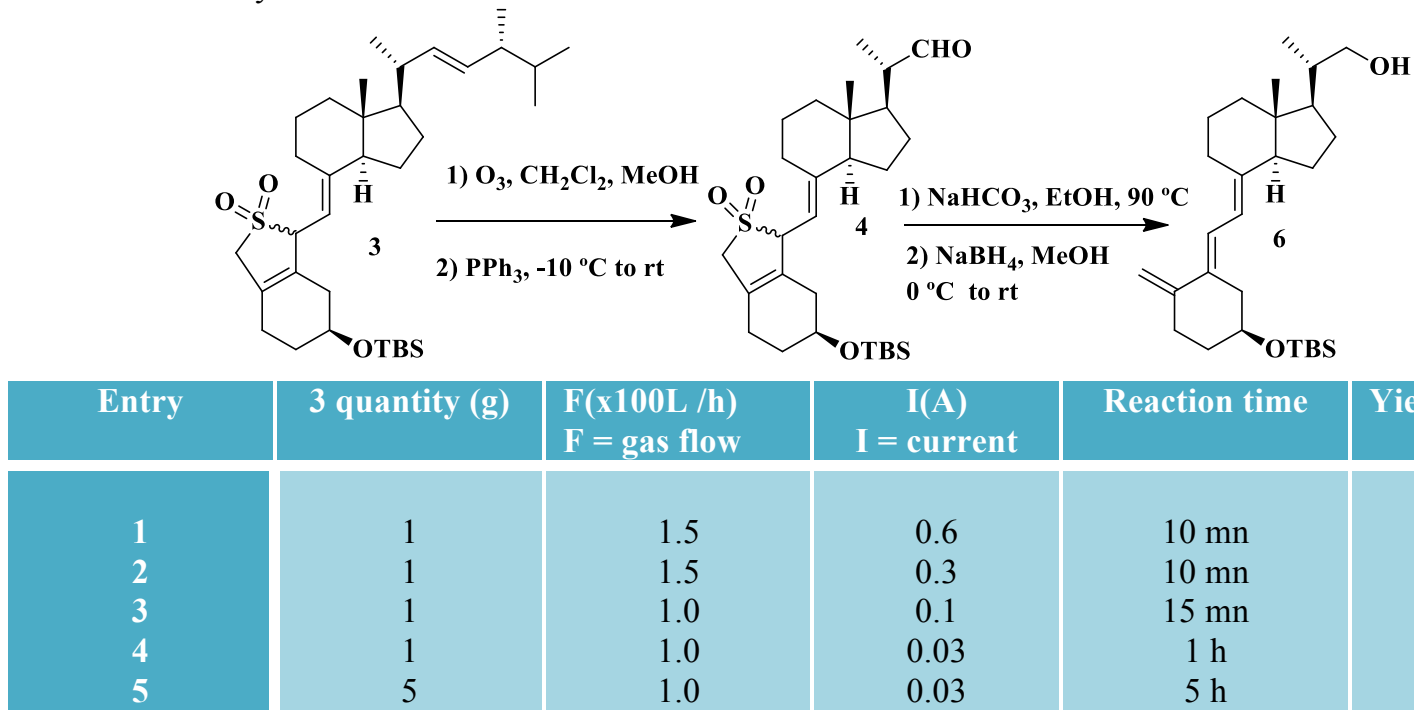

I(A)
$\mathrm{I}=$ curr


0.6
0.3
0.1
0.03
0.03

Reaction time (from 3)

\begin{tabular}{l|l}
1 & 1.5 \\
1 & 1.5 \\
1 & 1.0 \\
1 & 1.0 \\
5 & 1.0
\end{tabular}


The optimized reaction conditions for running the ozonolysis of $\mathbf{3}$ were as described in entries 4 and 5 .

The time necessary for the ozonolysis to be completed was substrate dependant ( $1 \mathrm{~h}$ to ozonolyze $1 \mathrm{~g}$ of substrate 3 ). Aldehyde 4 was unstable and was immediately converted to alcohol $\mathbf{6}$ by thermal chelotropic extrusion of sulfur dioxide $\left(\mathrm{SO}_{2}\right)$ in the presence of sodium bicarbonate $\left(\mathrm{NaHCO}_{3}\right)$ followed by sodium borohydride reduction of the intermediate aldehyde 5. Alcohol 6 was obtained in $85 \%$ overall yield (3 steps).

Reaction of alcohol $\mathbf{6}$ with acetic anhydride gave $96 \%$ yield of acetate 7 which was hydroxylated at C1 with selenium dioxide in the presence of 4methylmorpholine $\mathrm{N}$-oxide (NMO) to afford allylic alcohol 8 in $60 \%$ yield. The latter was silylated, giving $88 \%$ yield of acetate 9 . Reaction of the latter with potassium carbonate $\left(\mathrm{K}_{2} \mathrm{CO}_{3}\right)$ in methanol afforded key intermediate $\mathbf{1 0}$ with the $5-(E)$ triene system.

The overall yield of intermediate $\mathbf{1 0}$ from vitamin $\mathrm{D}_{2}$ was $39 \%$. Worth mentioning that the synthesis of
10 could be carried out in multigrams quantities and the compound could be stored in the fridge during months without alteration. Compound $\mathbf{1 0}$ is more stable than its $5-(Z)$ isomer and can be further elaborated in order to introduce the desired side chain.

The advantages of this present approach compared to Calverley and Choudhry's approaches are: 1) Only one $\mathrm{SO}_{2}$ triene protection is needed, hence one $\mathrm{SO}_{2}$ extrusion. 2) The ozonolysis of the side chain is carried out before the C-1 hydroxylation. 3) For the synthesis of $\mathbf{1 0}$ from vitamin $\mathrm{D}_{2}$, we found an overall yield of $39 \%$ which is a bit better then $37 \%$ calculated using Calverley's procedure.

We anticipated that intermediate $\mathbf{1 0}$ could lead to calcitriol analogues 15, 16, and $\mathbf{1 7}$ using a "Click" chemistry approach ${ }^{5}$ between azide $\mathbf{1 1}$ and commercially available alkynes 12,13 , and 14 . Our retrosynthetic basis is outlined in Scheme 2.<smiles>C#CC(C)(O)C#CC(C)(O)C(C)(C)CC</smiles>

Scheme 2. Retrosynthetic analysis of analogues 15, 16 and 17

Accordingly compounds $\mathbf{1 5 - 1 7}$ were prepared as outlined in Scheme 3. 


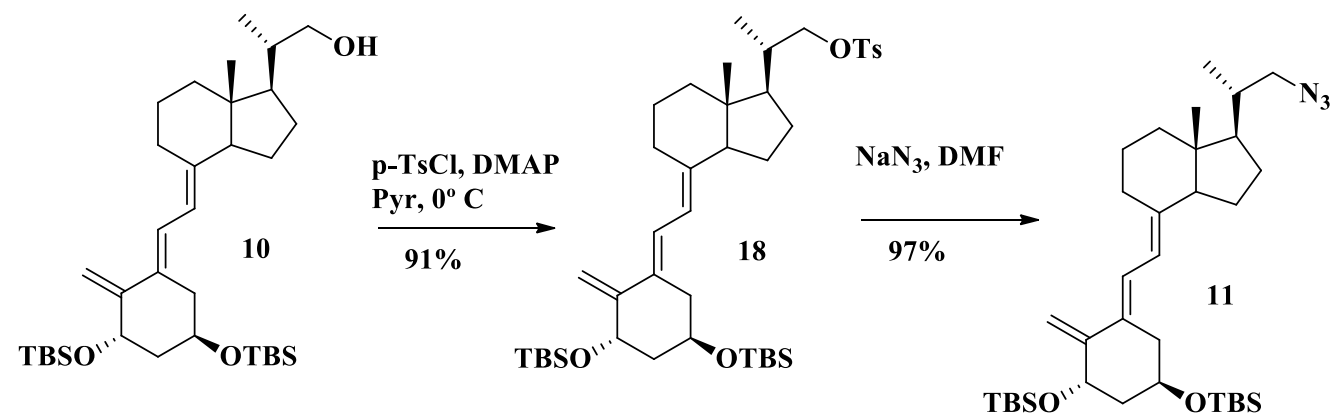

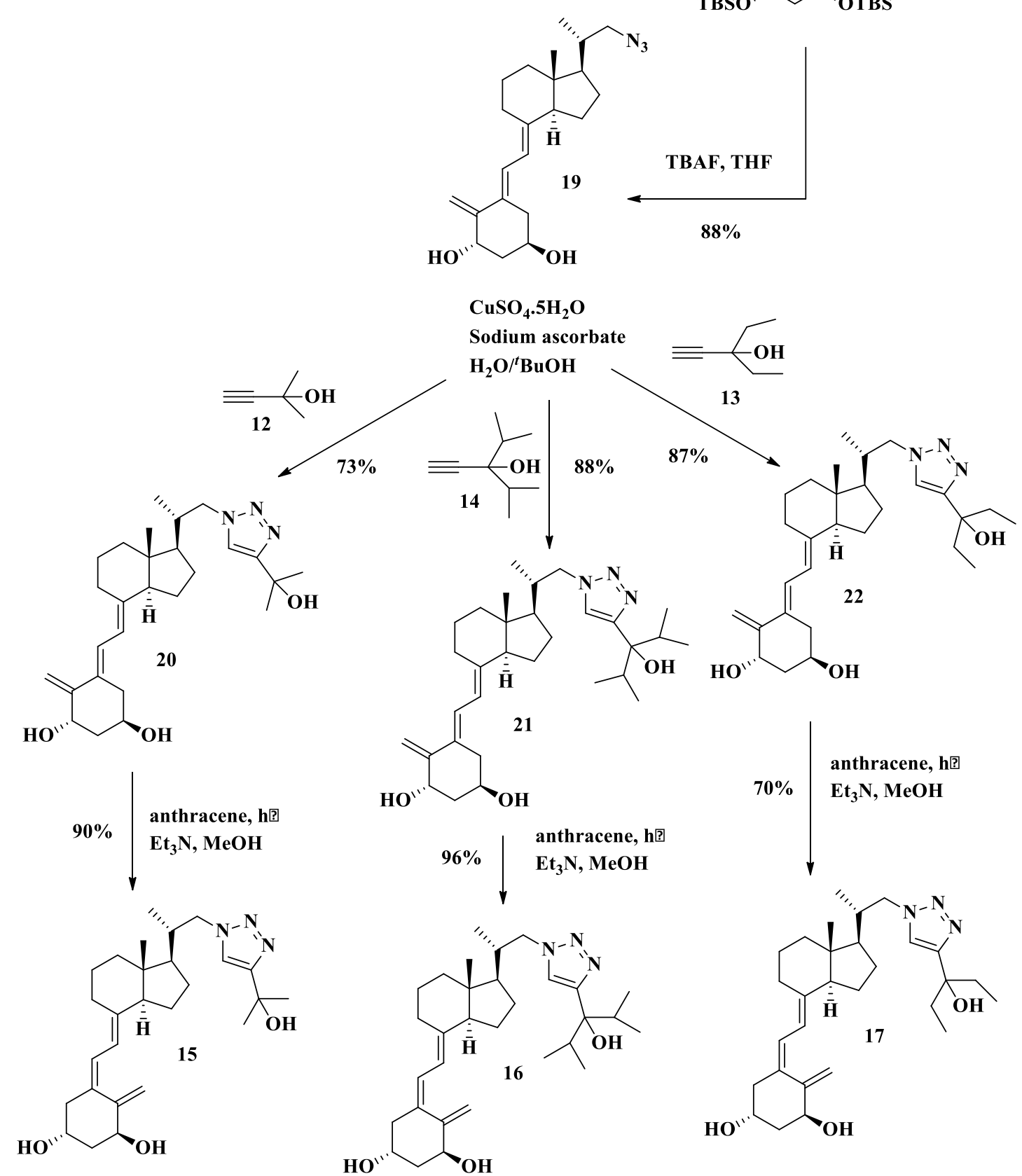

Scheme 3. Synthesis of analogues 15, 16 and 17

Tosylation of alcohol $\mathbf{1 0}$ followed by displacement of the C-22 tosylate of $\mathbf{1 8}$ with sodium azide, led to key azide $\mathbf{1 1}$ in $88 \%$ overall yield. Removal of the silyl protecting groups of $\mathbf{1 1}$ afforded azide 19 which underwent a [3+2]-cycloaddition ${ }^{5}$ with alkynes 12, 13 and $\mathbf{1 4}$ to afford triazoles 20, 21 and 22 in 73, 88 and $87 \%$ yields respectively.
Computational studies carried out by Sharpless and co-workers, ${ }^{6}$ proved that the exclusive regioselectivity of the triazole formation could be explained by a stepwise mechamism involving unprecedented matallacycle intermediates (Figure 2). 


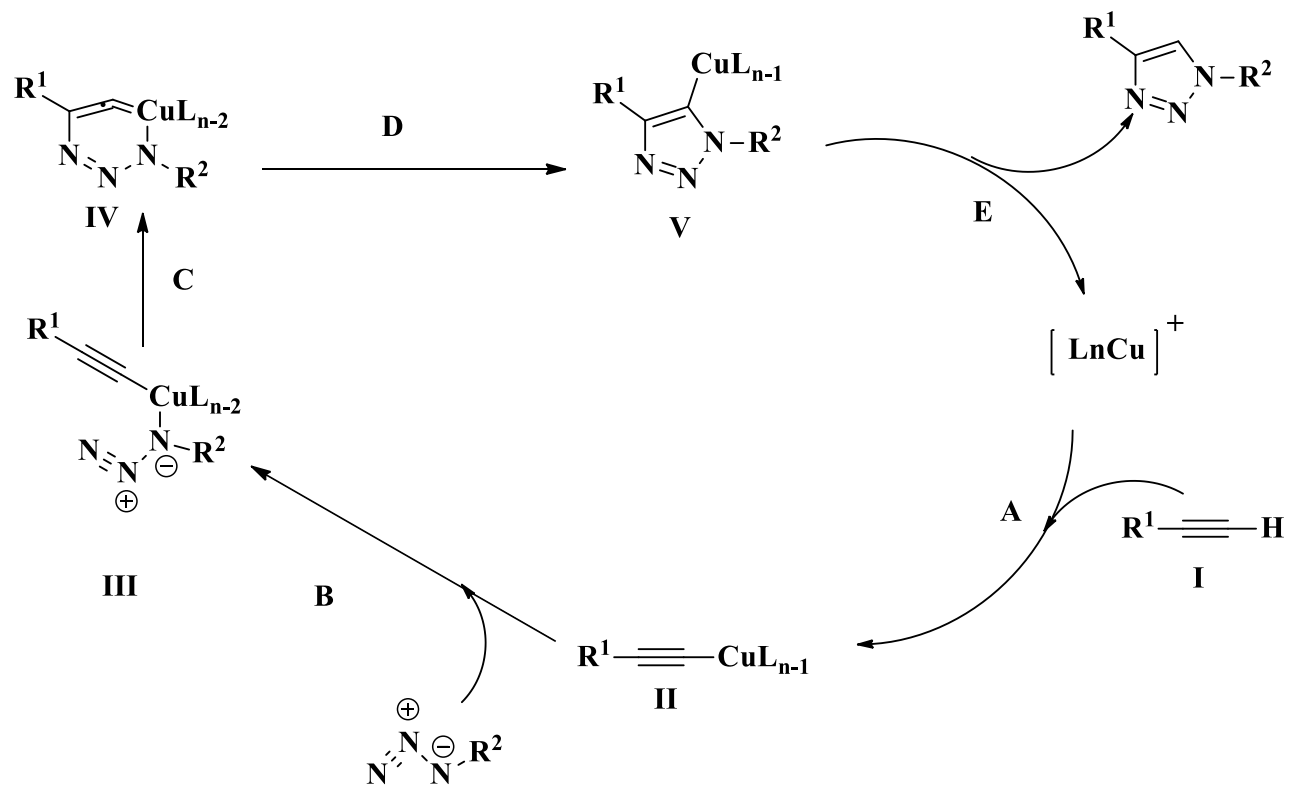

Figure 2. Sharpless proposed mechanism for the formation of 1,4-disubstituted 1,2,3-triazoles

Photosensitized isomerization of 20, 21 and 22 using anthracene as triplet sensitizer afforded target Vitamin D analogues 15, 16 and 17 in $90 \%, 96 \%$ and $70 \%$ yields respectively.

\section{Conclusion}

In conclusion, we have improved the method described by Calverley and Choudhry for the large scale synthesis of a late-stage intermediate which leads to a straightforward access to some calcitriol analogues with a triazole ring in their side chain. The use of intermediates mentioned above to access new calcitriol analogues is underway in our laboratory. The preliminary results of the biological activity of some of our azavitamin D analogues showed that they had less calcemic effect than calcitriol. These results as well as the activities of the whole series of the synthesized analogues will be published in due time after patent protection.

\section{Acknowledgements}

This work was supported financially by the Xunta de Galicia (project CN 2012/184).The work of the NMR, SC-XRD and MS divisions of the research support services of the University of Vigo (CACTI) is also gratefully acknowledged. A.F; M.S.; M.G. and O.D. thank the University Cheikh Anta Diop (Dakar, Sénégal) for financial support for a research stay at the University of Vigo.

\section{Experimental Section}

\section{General Procedures}

Solvents were purified and dried by standard procedures. Flash chromatography was performed on silicagel (Merck 60, 230-400 mesh). Analytical TLC was performed on plates precoated with silica gel
(Merck 60 F254, $0.25 \mathrm{~mm}$ ). Melting points were obtained using a Gallenkamp apparatus and are uncorrected. Optical rotations were obtained using a Jasco P-2000 polarimeter. IR spectra were obtained using a Jasco FT/IR-6100 Type A spectrometer. ${ }^{1} \mathrm{H}$ NMR (400 MHz) and ${ }^{13} \mathrm{C}$ NMR (100 MHz) spectra were recorded on a Bruker ARX-400 spectrometer using TMS as the internal standard; chemical shifts $(\delta)$ are quoted in ppm and coupling constants $(\mathrm{J})$ in Hz. Mass spectrometry (MS and HRMS) was carried out using a Hewlett-Packard 5988A spectrometer. The reactions were carried out protecting the glassware from light using aluminum foil.

(6S)-6-((tert-butyldimethylsilyl)oxy)-1-((E)((3aS,7aR)-1-((2R,5R,E)-5,6-dimethylhept-3-en-2yl)-7a-methylhexahydro- $1 \mathrm{H}$-inden-4(2H)ylidene)methyl)-1,3,4,5,6,7hexahydrobenzo[c]thiophene 2,2-dioxide (3)

In a three neckround-bottom flask at $-25{ }^{\circ} \mathrm{C}$ was condensed $\mathrm{SO}_{2}(100 \mathrm{~mL}, 2.00 \mathrm{~mol})$ and a solution of vitamin $\mathrm{D}_{2}(100 \mathrm{~g}, 0.25 \mathrm{~mol})$ in $\mathrm{CH}_{2} \mathrm{Cl}_{2}(250 \mathrm{~mL})$ was added via cannula. At the end of the addition the orange mixture was stirred at $-10^{\circ} \mathrm{C}$ for $90 \mathrm{~min}$ and allowed to reach room temperature, thus removing excess $\mathrm{SO}_{2}$. The solvent was evaporated and the residue was dissolved in $\mathrm{CH}_{2} \mathrm{Cl}_{2}$ and cooled to $5{ }^{\circ} \mathrm{C}$. Imidazole (22.5 g, $0.33 \mathrm{~mol})$, TBSCl $(50 \mathrm{~g}, 0.33$ mol) and a catalytic amount of DMAP were added to the mixture which was stirred overnight, reaching room temperature. $\mathrm{H}_{2} \mathrm{O}(100 \mathrm{~mL})$ was added and the aqueous phase was extracted with $\mathrm{CH}_{2} \mathrm{Cl}_{2}(3 \times 25$ $\mathrm{mL})$. The combined organic phases were washed with brine $(25 \mathrm{~mL})$ and dried. Solvent evaporation afforded $140 \mathrm{~g}(96 \%)$ of known compound $\mathbf{3}^{4 \mathrm{~b}}$. 


\section{(2S)-2-((3aS,7aR, E)-4-((E)-2-((S)-5-((tert- butyldimethylsilyl)oxy)-2- methylenecyclohexylidene)ethylidene)-7a- methyloctahydro-1H-inden-1-yl)propan-1-ol (6)}

A solution of $3(5 \mathrm{~g}, 8.7 \mathrm{mmol})$ in $\mathrm{MeOH}$ $(66 \mathrm{~mL})$ and $\mathrm{CH}_{2} \mathrm{Cl}_{2}(170 \mathrm{~mL})$ was subjected to ozonolysis using the best conditions described in Table 1 and after $5 \mathrm{~h}$ at $-78^{\circ} \mathrm{C}$ the mixture was allowed to reach $-10^{\circ} \mathrm{C} . \mathrm{PPh}_{3}(3 \mathrm{~g}, 11.5 \mathrm{mmol})$ was added and stirring was continued for $30 \mathrm{~min}$. The mixture was allowed to reach $0{ }^{\circ} \mathrm{C}$ and an aqueous saturated solution of $\mathrm{NaHCO}_{3}(40 \mathrm{~mL})$ was added. After extraction with $\mathrm{CH}_{2} \mathrm{Cl}_{2}(3 \times 100 \mathrm{~mL})$, the combined organic phases were washed with brine (3 x $50 \mathrm{~mL})$, dried $\left(\mathrm{Na}_{2} \mathrm{SO}_{4}\right)$, filtered and concentrated to give $4.43 \mathrm{~g}$ of a residue (aldehyde 4) which was used for the next reaction without further purification. The residue was dissolved in ethanol (45 mL) and $\mathrm{NaHCO}_{3}(4.43 \mathrm{~g})$ was added and the mixture was refluxed for $5 \mathrm{~h}$. The solvent was rotatory evaporated affording a residue which was dissolved in $\mathrm{CH}_{2} \mathrm{Cl}_{2}(25 \mathrm{~mL})$ and filtered in order to remove excess $\mathrm{NaHCO}_{3}$. To the filtrate was added brine $(50 \mathrm{~mL})$. After extraction with $\mathrm{CH}_{2} \mathrm{Cl}_{2}(3 \times 25$ $\mathrm{mL})$, the combined organic phases were dried $\left(\mathrm{Na}_{2} \mathrm{SO}_{4}\right)$, filtered and concentrated to afford a residue (compound 5) which was used for the next reaction without further purification. The residue was dissolved in $\mathrm{MeOH}(45 \mathrm{~mL})$ and cooled to $0{ }^{\circ} \mathrm{C}$. $\mathrm{NaBH}_{4} \quad(400 \mathrm{mg}, \quad 10.44 \mathrm{mmol})$ was added portionwise to the mixture and stirring was continued for $15 \mathrm{~min}$. $\mathrm{H}_{2} \mathrm{O}(50 \mathrm{~mL})$ was added and the product extracted with $\mathrm{CH}_{2} \mathrm{Cl}_{2}(3 \times 50 \mathrm{~mL})$. The combined organic phases were dried $\left(\mathrm{Na}_{2} \mathrm{SO}_{4}\right)$, filtered and concentrated to afford a residue which was chromatographed on silica gel using $10 \%$ EtOAc/Hexane as eluent, affording $3.3 \mathrm{~g}(85 \%, 3$ steps) of alcohol 6, as a white solid, M.p.: $53-55^{\circ} \mathrm{C}$, $\mathrm{Rf}=0.59$ (30\% EtOAc/Hexane);

${ }^{1} \mathbf{H}-\mathbf{N M R}\left(\mathbf{C D C l}_{3}, \boldsymbol{\delta}\right): 4.74(1 \mathrm{H} ; \mathrm{d} ; \mathrm{J}=10,08 \mathrm{~Hz}$; H-7); 4.58 (1H; d; J=9,6 Hz; H-6); 3.92 (1H; s; H-3); 3.50 (2H; m; H-22);3.32 (2H; s; $\left.\mathrm{CH}_{2}-19\right) ; 2.85$ $(1 \mathrm{H}, \mathrm{m}) ; 2.43(2 \mathrm{H}, \mathrm{m}), 2.36(2 \mathrm{H}, \mathrm{m}) ; 2.29(1 \mathrm{H}, \mathrm{m})$; $2.06(1 \mathrm{H}, \mathrm{m}) ; 1.98(1 \mathrm{H}, \mathrm{m}) ; 1.86(2 \mathrm{H}, \mathrm{m}), 1.65(7 \mathrm{H}$, m); $1.36(3 \mathrm{H}, \mathrm{m}), 0.99\left(3 \mathrm{H} ; \mathrm{d} ; \mathrm{J}=6,48 \mathrm{~Hz} ; \mathrm{CH}_{3}-21\right)$; $0.82\left(9 \mathrm{H} ; \mathrm{s} ;\right.$ tert-BuSi); $0.53\left(3 \mathrm{H} ; \mathrm{s} ; \mathrm{CH}_{3}-18\right)$; $0.01\left(3 \mathrm{H} ; \mathrm{s} ; \mathrm{CH}_{3}-\mathrm{TBS}\right) ; 0.00$ (3H; s; $\left.\mathrm{CH}_{3}-\mathrm{TBS}\right)$;

${ }^{13} \mathbf{C}-N M R\left(\mathbf{C D C l}_{3}, \boldsymbol{\delta}\right)$ : 150.3 (C-8); 131.1 (C-5); 127.1 (C-10); 116.1 (C-7); $110.2\left(\mathrm{CH}_{2}-19\right) ; 68.0$ $\left(\mathrm{CH}_{2}-22\right) ; 67.2(\mathrm{CH}-3$ y $\mathrm{CH}-6) ; 58.5\left(\mathrm{CH}_{2}\right) ; 56.2$ (C-14); 53.1 (C-17); 46.0 (C-13); $40.3\left(\mathrm{CH}_{2}\right) ; 39.4$ $(\mathrm{C}-20) ; 34.5\left(\mathrm{CH}_{2}\right) ; 31.4\left(\mathrm{CH}_{2}\right) ; 29.8\left(\mathrm{CH}_{2}\right) ; 27.4$ $\left(\mathrm{CH}_{2}\right) ; 26.2\left(\mathrm{CH}_{3}\right.$-terc-BuSi); $25.0 \quad\left(\mathrm{CH}_{2}\right) ; 23.9$ $\left(\mathrm{CH}_{2}\right) ; 22.6\left(\mathrm{CH}_{2}\right) ; 18.5(\mathrm{C}-$ tert-Bu); $17.3(\mathrm{C}-21)$; 12.1 (C-18); -4.6 ( $\left.\mathrm{CH}_{3}-\mathrm{TBS}\right) ;-4.7\left(\mathrm{CH}_{3}-\mathrm{TBS}\right)$;

MS (m/z (\%)): $445.22\left(\mathrm{M}^{+}+1,20\right) ; 311.13$ (32); 281.09 (20); 267.08 (21); 209.10 (41); 193.15 (100);

HRMS: Calcdfor $\mathrm{C}_{28} \mathrm{H}_{48} \mathrm{O}_{2} \mathrm{Si}$ : 445.3502, found: 445.3508.

\author{
(2S)-2-((3aS,7aR, E)-4-((E)-2-((S)-5-((tert- \\ butyldimethylsilyl)oxy)-2- \\ methylenecyclohexylidene)ethylidene)-7a- \\ methyloctahydro-1H-inden-1-yl)propyl acetate \\ (7)
}

To a solution of alcohol $6(3.0 \mathrm{~g}, 6.75 \mathrm{mmol})$ in $\mathrm{CH}_{2} \mathrm{Cl}_{2}(30 \mathrm{~mL})$ were added pyr $(1.2 \mathrm{~mL}, 14.8$ $\mathrm{mmol}), \mathrm{Ac}_{2} \mathrm{O}(0.7 \mathrm{~mL}, 7.43 \mathrm{mmol})$ and a catalytic amount of DMAP. The mixture was stirred for $2 \mathrm{~h}$ at room temperature and cooled to $0{ }^{\circ} \mathrm{C}$ before adding an aqueous saturated solution of $\mathrm{NH}_{4} \mathrm{Cl}(30 \mathrm{~mL})$. After extraction with $\mathrm{CH}_{2} \mathrm{Cl}_{2}(3 \times 40 \mathrm{~mL})$, the combined organic phases were washed with an aqueous saturated solution of $\mathrm{CuSO}_{4}(3 \times 40 \mathrm{~mL})$, dried $\left(\mathrm{Na}_{2} \mathrm{SO}_{4}\right)$, filtered and concentrated to afford a residue which was chromatographed on silica gel using 5\% EtOAc/Hexane affording $2.9 \mathrm{~g}(94 \%)$ of acetate 7 , as a yellowish oil; $\mathrm{Rf}=0.66(10 \%$ EtOAc/Hexane);

${ }^{1} \mathbf{H}-\mathbf{N M R}\left(\mathbf{C D C l}_{3}, \boldsymbol{\delta}\right): 6.50(1 \mathrm{H} ; \mathrm{d} ; \mathrm{J}=11,45 \mathrm{~Hz} ; \mathrm{H}-6)$; 5.88 (1H; d; J=11,50; H-7); 4.94 (1H; s; H-19); 4.65 $(1 \mathrm{H}$; s; H-19); 4.10 (1H, dd, J=3.8 y $7.4 \mathrm{~Hz}, \mathrm{H}-22)$; $3.86(1 \mathrm{H}, \mathrm{t}, \mathrm{J}=3.8 \mathrm{~Hz}, \mathrm{H}-3) ; 3.81(1 \mathrm{H}, \mathrm{dd}, \mathrm{J}=3.8$ y 7.4 $\mathrm{Hz}, \mathrm{H}-22) ; 2.85(1 \mathrm{H}, \mathrm{m}) ; 2.68(1 \mathrm{H}, \mathrm{m}) ; 2.52(1 \mathrm{H}$, m); $2.22(2 \mathrm{H}, \mathrm{m}) ; 2.15(1 \mathrm{H}, \mathrm{m}) ; 2.07\left(3 \mathrm{H}, \mathrm{s}, \mathrm{CH}_{3}-\right.$ Ac); $2.00(1 \mathrm{H}, \mathrm{m}) ; 1.86(2 \mathrm{H}, \mathrm{m}) ; 1.65(7 \mathrm{H}, \mathrm{m}) ; 1.35$ $(3 \mathrm{H}, \mathrm{m}) ; 1.05\left(3 \mathrm{H} ; \mathrm{d} ; \mathrm{J}=6.54 \mathrm{~Hz} ; \mathrm{CH}_{3}-21\right) ; 0.89$ (9H; s; tert-BuSi); 0.59 (3H; s; $\left.\mathrm{CH}_{3}-18\right) ; 0.08(3 \mathrm{H}, \mathrm{s}$, $\mathrm{CH}_{3}$-TBS); 0.07(3H, s, $\mathrm{CH}_{3}$-TBS);

${ }^{13} \mathbf{C}-N M R\left(\mathbf{C D C l}_{3}, \boldsymbol{\delta}\right): 171.4(\mathrm{C}=\mathrm{O}) ; 150.0(\mathrm{C}-10)$; 143.2 (C-8); 136.5 (C-5); 119.9 (CH-6); 116.2 (CH$7) ; 107.6\left(\mathrm{CH}_{2}-19\right) ; 69.6(\mathrm{CH}-3) ; 69.5\left(\mathrm{CH}_{2}-22\right)$; 56.1 (CH-14); 53.1 (CH-17); 45.9 (C-13); 40.3 $\left(\mathrm{CH}_{2}\right) ; 37.5\left(\mathrm{CH}_{2}\right) ; 36.2(\mathrm{CH}-20) ; 35.2\left(\mathrm{CH}_{2}\right) ; 31.2$ $\left(\mathrm{CH}_{2}\right) ; 28.9\left(\mathrm{CH}_{2}\right) ; 27.2\left(\mathrm{CH}_{2}\right) ; 26.0\left(\mathrm{CH}_{3}\right.$-tertBuSi); $23.5\left(\mathrm{CH}_{2}\right) ; 22.3\left(\mathrm{CH}_{2}\right) ; 23.0\left(\mathrm{CH}_{3}-\mathrm{Ac}\right) ; 18.2$ (C-tert-BuSi); $17.3\left(\mathrm{CH}_{3}-21\right) ; 12.1\left(\mathrm{CH}_{3}-18\right) ;-4,6$ ( $\left.\mathrm{CH}_{3}-\mathrm{TBS}\right)$;

MS (m/z (\%)): $487.33\left(\mathrm{M}^{+}+1,12\right) ; 486.33\left(\mathrm{M}^{+}, 23\right)$; 295.20 (21); 193.15 (100); 171.21 (32);

HRMS: Calcd for $\mathrm{C}_{30} \mathrm{H}_{50} \mathrm{O}_{3} \mathrm{Si}$ : 486.3529, found: 486.3518 .

\section{(2S)-2-((3aS,7aR, E)-4-((E)-2-((3S,5R)-5-((tert- butyldimethylsilyl)oxy)-3-hydroxy-2- methylenecyclohexylidene)ethylidene)-7a- methyloctahydro-1H-inden-1-yl)propyl acetate (8)}

A solution of $\mathrm{SeO}_{2}(0.7 \mathrm{~g}, 6.32 \mathrm{mmol})$ in $\mathrm{MeOH}$ $(50 \mathrm{~mL})$ was refluxed for $45 \mathrm{~min}$. A solution of acetate $7(2.18 \mathrm{~g}, 4.47 \mathrm{mmol})$ in $\mathrm{CH}_{2} \mathrm{Cl}_{2}(52 \mathrm{~mL})$ was also refluxed for $15 \mathrm{~min}$ before adding it via cannula to the previous solution of $\mathrm{SeO}_{2}$. After the addition, the mixture was refluxed for $2 \mathrm{~h}$ and allowed to reach room temperature. $\mathrm{H}_{2} \mathrm{O}(10 \mathrm{~mL})$ was added and the product extracted with $\mathrm{CH}_{2} \mathrm{Cl}_{2}(3 \times 25 \mathrm{~mL})$. The combined organic phases were dried $\left(\mathrm{Na}_{2} \mathrm{SO}_{4}\right)$, filtered and concentrated to afford a residue which was chromatographed on silica gel using $10 \%$ EtOAc/Hexane affording $1.37 \mathrm{~g}(61 \%)$ of alcohol $\mathbf{8}$, 
as a white solid, M.p.: $44^{\circ} \mathrm{C}, \mathrm{Rf}=0.42(20 \%$ EtOAc/Hexane);

${ }^{1} \mathbf{H}-\mathbf{N M R}\left(\mathbf{C D C l}_{3}, \boldsymbol{\delta}\right): 6.45(1 \mathrm{H}, \mathrm{d}, \mathrm{J}=11.4 \mathrm{~Hz} ; \mathrm{H}-6)$; $5.80(1 \mathrm{H}, \mathrm{d}, \mathrm{J}=11.4 \mathrm{~Hz} ; \mathrm{H}-7) ; 4.42(1 \mathrm{H}, \mathrm{s}, \mathrm{H}-19)$; 4,12 (1H, s, H-19); 4,01 (1H, dd, J=7,43 y 3,24 Hz, $\mathrm{H}-22) ; 3,74(1 \mathrm{H}, \mathrm{dd}, \mathrm{J}=7,5$ y $3,20 \mathrm{~Hz}, \mathrm{H}-22) ; 2.75$ $(2 \mathrm{H}, \mathrm{m}) ; 2.43(1 \mathrm{H}, \mathrm{m}) ; 2.33(1 \mathrm{H}, \mathrm{m}) ; 1,98(3 \mathrm{H}, \mathrm{s}$, $\left.\mathrm{CH}_{3}-\mathrm{Ac}\right) ; 1.86(4 \mathrm{H}, \mathrm{m}) ; 1.62(4 \mathrm{H}, \mathrm{m}) ; 1.46(3 \mathrm{H}, \mathrm{m})$; $1.22(2 \mathrm{H}, \mathrm{m}) ; 0,96\left(3 \mathrm{H}, \mathrm{d}, \mathrm{J}=6,6 \mathrm{~Hz} ; \mathrm{CH}_{3}-21\right) ; 0,79$ $\left(9 \mathrm{H}, \mathrm{s}\right.$, tert-BuSi); 0,50(3H, s, $\left.\mathrm{CH}_{3}-18\right) ; 0,00(6 \mathrm{H}, \mathrm{s}$, $\mathrm{CH}_{3}$-TBS);

${ }^{13} \mathbf{C}-N M R\left(\mathrm{CDCl}_{3}, \boldsymbol{\delta}\right): 171.8(\mathrm{C}=\mathrm{O}) ; 153.5(\mathrm{C}-10)$; 144.0 (C-8); 135.0 (C-5); 122.6 (C-6); 116.8 (C-7); $108.1\left(\mathrm{CH}_{2}-19\right) ; 70.9$ (C-3); $69.8\left(\mathrm{CH}_{2}-22\right) ; 67.2$ (C-1); 56.5 (C-14); 53.5 (C-17); 46.4 (C-13); $43.3\left(\mathrm{CH}_{2}\right) ; 40.7\left(\mathrm{CH}_{2}\right) ; 37.3\left(\mathrm{CH}_{2}\right) ; 36.5(\mathrm{C}-20)$; $29.4\left(\mathrm{CH}_{2}\right) ; 27.5\left(\mathrm{CH}_{2}\right) ; 26.2\left(\mathrm{CH}_{3}\right.$-terc-BuSi); 23.9 $\left(\mathrm{CH}_{2}\right) ; 22.7\left(\mathrm{CH}_{2}\right) ; 21.4\left(\mathrm{CH}_{3}-\mathrm{Ac}\right) ; 18.5$ (C-tert$\mathrm{BuSi}) ; 17.7$ (C-21); 12.5 (C-18); -4.3 ( $\mathrm{CH}_{3}$-TBS);

MS (m/z (\%)): $503.25\left(\mathrm{M}^{+}+1,18\right) ; 502.34\left(\mathrm{M}^{+}, 23\right)$; 307.16 (20); 171.21 (32);

HRMS: Calcdfor $\mathrm{C}_{30} \mathrm{H}_{50} \mathrm{O}_{4} \mathrm{Si}:$ 502.3478, found: 502.3495 .

(2S)-2-((3aS,7aR,E)-4-((E)-2-((3S,5R)-3,5-bis((tertbutyldimethylsilyl)oxy)-2-

methylenecyclohexylidene)ethylidene)-7a-

methyloctahydro-1H-inden-1-yl)propyl acetate (9)

To a solution of alcohol $8(1.90 \mathrm{~g}, 3.76 \mathrm{mmol})$ in $\mathrm{CH}_{2} \mathrm{Cl}_{2}(10 \mathrm{~mL})$, at $0{ }^{\circ} \mathrm{C}$ was added imidazole (343 $\mathrm{mg}, 5.0 \mathrm{mmol})$, TBSCl $(760 \mathrm{mg}, 5.0 \mathrm{mmol})$ and a catalytic amount of DMAP and the mixture was left stirring at room temperature for $5 \mathrm{~h} . \mathrm{H}_{2} \mathrm{O}(10 \mathrm{~mL})$ was added and the product extracted with $\mathrm{CH}_{2} \mathrm{Cl}_{2}(3$ x $20 \mathrm{~mL}$ ). The combined organic phases were washed with brine $(20 \mathrm{~mL})$, dried $\left(\mathrm{Na}_{2} \mathrm{SO}_{4}\right)$, filtered and concentrated to afford a residue which was chromatographed on silica gel using $3 \%$ EtOAc/Hexane as solvent, affording $2.1 \mathrm{~g}(90 \%)$ of acetate 9 , as a white solid, M.p.: $77-80{ }^{\circ} \mathrm{C}, \mathrm{Rf}=0.51$ (20\% EtOAc/Hexane);

${ }^{1} \mathbf{H}-\mathbf{N M R}\left(\mathbf{C D C l}_{3}, \boldsymbol{\delta}\right): 6.40(1 \mathrm{H}, \mathrm{d}, \mathrm{J}=11.32 \mathrm{~Hz} ; \mathrm{CH}-$ 6); $5.78(1 \mathrm{H}, \mathrm{d}, \mathrm{J}=11.16 \mathrm{~Hz} ; \mathrm{CH}-7) ; 4.92(1 \mathrm{H}, \mathrm{s}$; $\left.\mathrm{CH}_{2}-19\right) ; 4.88\left(1 \mathrm{H}, \mathrm{s} ; \mathrm{CH}_{2}-19\right) ; 4.48(1 \mathrm{H}, \mathrm{d}$; $\mathrm{J}=4.7 \mathrm{~Hz} ; \mathrm{CH}-3) ; 4.16(1 \mathrm{H}, \mathrm{s} ; \mathrm{CH}-1) ; 4.05(1 \mathrm{H}$, dd; $\mathrm{J}=3.25 \mathrm{~Hz} ; \mathrm{J}=7.34 \mathrm{~Hz} ; \mathrm{H}-22) ; 3.74\left(1 \mathrm{H}, \mathrm{dd} ; \mathrm{J}_{1}=3.22\right.$ $\left.\mathrm{Hz} ; \mathrm{J}_{2}=7.39 \mathrm{~Hz} ; \mathrm{H}-22\right) ; 2.86(1 \mathrm{H}, \mathrm{m}) ; 2.55(1 \mathrm{H}, \mathrm{m})$; $2.22(1 \mathrm{H}, \mathrm{m}) ; 1.98\left(3 \mathrm{H}, \mathrm{s} ; \mathrm{CH}_{3}-\mathrm{Ac}\right) ; 1.95(1 \mathrm{H}, \mathrm{m})$; $1.85(3 \mathrm{H}, \mathrm{m}) ; 1.65(4 \mathrm{H}, \mathrm{m}) ; 1.55(3 \mathrm{H}, \mathrm{m}) ; 1.33(3 \mathrm{H}$, $\mathrm{m}) ; 1.01\left(3 \mathrm{H}, \mathrm{s}, \mathrm{CH}_{3}-21\right) ; 0.84\left(9 \mathrm{H}, \mathrm{s} ; \mathrm{CH}_{3}\right.$-tert$\mathrm{BuSi}) ; 0.80\left(9 \mathrm{H}, \mathrm{s} ; \mathrm{CH}_{3}\right.$-tert-BuSi); 0.51(3H, s; $\mathrm{CH}_{3}-$ 18); $0.00\left(12 \mathrm{H}, \mathrm{s} ; \mathrm{CH}_{3}\right.$-TBS $)$;

${ }^{13} \mathbf{C}-\mathbf{N M R}\left(\mathrm{CDCl}_{3}, \boldsymbol{\delta}\right)$ : $171.6(\mathrm{C}=\mathrm{O}) ; 154.0(\mathrm{C}-10)$; 143.1 (C-8); 136.0 (C-5); $122.0(\mathrm{CH}-6) ; 117.0(\mathrm{CH}-$ $7) ; 107.0\left(\mathrm{CH}_{2}-19\right) ; 70.6(\mathrm{CH}-3) ; 69.8\left(\mathrm{CH}_{2}-22\right)$; 67.6 (CH-1); 56.5 (CH-14); 53.5 (CH-17); 46.4 $(\mathrm{C}-13) ; 44.3\left(\mathrm{CH}_{2}\right) ; 40.8\left(\mathrm{CH}_{2}\right) ; 36.9\left(\mathrm{CH}_{2}\right) ; 36.5$ (CH-20); $29.3\left(\mathrm{CH}_{2}\right) ; 27.5\left(\mathrm{CH}_{2}\right) ; 26.3\left(\mathrm{CH}_{3}\right.$-tercBuSi); $23.8\left(\mathrm{CH}_{2}\right) ; 22.7\left(\mathrm{CH}_{2}\right) ; 21.3\left(\mathrm{CH}_{3}-\mathrm{Ac}\right) ; 18.6$
(C-tert-BuSi); 18.4 (C-tert-BuSi); $17.7\left(\mathrm{CH}_{3}-21\right)$; $12.4\left(\mathrm{CH}_{3}-18\right) ;-4.4\left(\mathrm{CH}_{3}-\mathrm{TBS}\right) ;-4.5\left(\mathrm{CH}_{3}-\mathrm{TBS}\right)$;

MS (m/z (\%)): $617.44 \quad\left(\mathrm{M}^{+}+1,38\right) ; 616.42$ (23); 485.33 (52); 284.16 (100); 171.21 (32); 163.22 (20);

HRMS: Calcdfor $\mathrm{C}_{36} \mathrm{H}_{64} \mathrm{O}_{4} \mathrm{Si}_{2}:$ 616.4298, found: 616.4262

\section{(2S)-2-((3aS,7aR, E)-4-((E)-2-((3S,5R)-3,5- bis((tert-butyldimethylsilyl)oxy)-2- methylenecyclohexylidene)ethylidene)-7a- methyloctahydro-1H-inden-1-yl)propan-1-ol (10)}

To a solution of acetate $9(1.1 \mathrm{~g}, 1.83 \mathrm{mmol})$ in $\mathrm{MeOH}(30 \mathrm{~mL})$ at room temperature was added $\mathrm{K}_{2} \mathrm{CO}_{3}(500 \mathrm{mg}, 3.67 \mathrm{mmol})$ and the mixture was stirred for $10 \mathrm{~h}$. Excess $\mathrm{K}_{2} \mathrm{CO}_{3}$ was eliminated by firtration and $\mathrm{H}_{2} \mathrm{O}(100 \mathrm{~mL})$ was added to the filtrate and the product extracted with $\mathrm{CH}_{2} \mathrm{Cl}_{2}(3 \times 50 \mathrm{~mL})$. The combined organic phases were washed with brine $(3 \times 20 \mathrm{~mL})$, dried $\left(\mathrm{Na}_{2} \mathrm{SO}_{4}\right)$, filtered and concentrated to afford a residue which was chromatographed on silica gel using $3 \%$ EtOAc/Hexane as solvent, affording $990 \mathrm{mg}$ (94\%) of alcohol 10, as a white solid, M.p.: $110-113^{\circ} \mathrm{C}$, $\mathrm{Rf}=0.63(30 \%$ EtOAc/Hexane $)$;

${ }^{1} \mathbf{H}-\mathbf{N M R}\left(\mathbf{C D C l}_{3}, \boldsymbol{\delta}\right): 6.41(1 \mathrm{H}, \mathrm{d}, \mathrm{J}=11,3 \mathrm{~Hz} ; \mathrm{H}-6)$; $5.78(1 \mathrm{H}, \mathrm{d}, \mathrm{J}=11.3 ; \mathrm{CH}-7) ; 4.92(1 \mathrm{H}, \mathrm{s}, \mathrm{H}-19)$; $4.87(1 \mathrm{H}, \mathrm{s}, \mathrm{H}-19) ; 4.45(2 \mathrm{H}, \mathrm{m}, \mathrm{H}-22) ; 4.15(1 \mathrm{H}, \mathrm{m}$, $\mathrm{H}-1) ; 3.61$ (1H, m, H-3); 2.75 (1H, m); 2.45 (1H, m); $2.27(1 \mathrm{H}, \mathrm{m}) ; 1.95(2 \mathrm{H}, \mathrm{m}) ; 1.63(4 \mathrm{H}, \mathrm{m}) ; 1.51(3 \mathrm{H}$, m); $1.22(6 \mathrm{H}, \mathrm{m}) ; 1.00\left(3 \mathrm{H}, \mathrm{d}, \mathrm{J}=7,6 \mathrm{~Hz} ; \mathrm{CH}_{3}-21\right)$; $0.83(9 \mathrm{H}, \quad \mathrm{s}, \quad$ tert $-\mathrm{BuSi}) ; \quad 0.80(9 \mathrm{H}, \quad \mathrm{s}$, tert-BuSi$)$; $0.50\left(3 \mathrm{H}, \mathrm{s}, \mathrm{CH}_{3}-18\right) ; 0.00\left(12 \mathrm{H}, \mathrm{s}, \mathrm{CH}_{3}-\mathrm{TBS}\right)$;

${ }^{13} \mathbf{C}-\mathrm{NMR}\left(\mathrm{CDCl}_{3}, \boldsymbol{\delta}\right)$ : $154.0(\mathrm{C}-10) ; 143.5(\mathrm{C}-8)$; 135.9 (C-5); 122.1 (C-6); 116.9 (C-7); $107.1\left(\mathrm{CH}_{2}-\right.$ 19); 70.7 (C-3); $68.3\left(\mathrm{CH}_{2}-22\right) ; 67.6(\mathrm{C}-1) ; 56.6(\mathrm{C}-$ 14); 53.3 (C-17); 46.3 (C-13); $44.3\left(\mathrm{CH}_{2}\right) ; 40.8$ $\left(\mathrm{CH}_{2}\right) \quad 39.5 \quad(\mathrm{C}-20) ; \quad 37.0\left(\mathrm{CH}_{2}\right) ; \quad 29.3 \quad\left(\mathrm{CH}_{2}\right)$; $27.6\left(\mathrm{CH}_{2}\right) ; 26.3\left(\mathrm{CH}_{3}\right.$-tert-BuSi); $26.2 \quad\left(\mathrm{CH}_{3}\right.$-tert$\mathrm{BuSi}) ; 23.9\left(\mathrm{CH}_{2}\right) ; 22.7\left(\mathrm{CH}_{2}\right) ; 18.6$ (C-tert-BuSi); 18.5(C-tert-BuSi); 17.3 (C-21); 12.5 (C-18); -4,4 $\left(\mathrm{CH}_{3}-\mathrm{TBS}\right)$

MS (m/z (\%)): $575.36\left(\mathrm{M}^{+}+1,51\right) ; 442.25$ (77); 249.11 (45); 247.10 (27);

HRMS: Calcd for $\mathrm{C}_{34} \mathrm{H}_{62} \mathrm{O}_{3} \mathrm{Si}_{2}: 575.4316$, found: 575.4317

(S)-2-((1R,3aS,7aR, E)-4-((E)-2-((3S,5R)-3,5bis((tert-butyldimethylsilyl)oxy)-2methylenecyclohexylidene)ethylidene)-7amethyloctahydro-1H-inden-1-yl)propyl 4methylbenzenesulfonate (18).

To a solution of $\mathbf{1 0}(990 \mathrm{mg}, 1.72 \mathrm{mmol})$ in Py $(9 \mathrm{~mL})$ at $0{ }^{\circ} \mathrm{C}$ was added p-TsCl (660 mg, 3.44 mmol) and DMAP (c.c.). The mixture was stirred at this temperature for $9 \mathrm{~h}$, quenched with $\mathrm{NH}_{4} \mathrm{Cl}(10$ $\mathrm{mL}$ ), then allowed to reach room temperature. The product was extracted with EtOAc $(3 \times 15 \mathrm{~mL})$. The organic phase was washed with $\mathrm{CuSO}_{4}(3 \times 20 \mathrm{ml})$. 
After drying $\left(\mathrm{Na}_{2} \mathrm{SO}_{4}\right)$ and solvent evaporation, the residue was chromatographed on silicagel using $3 \%$ EtOAc-hexane as eluent, to afford $1.1 \mathrm{~g}$ of tosylate 18 [91\%, white solid; $\mathrm{Mp}=50-53{ }^{\circ} \mathrm{C}$; Rf: 0.87 (30\% EtOAc-hexane)].

${ }^{1}$ H-NMR $\left(\mathrm{CDCl}_{3}, \delta\right): 7.72(2 \mathrm{H}, \mathrm{d}, J=8.2 \mathrm{~Hz}, \mathrm{H}-\mathrm{Ts})$, $7.27(2 \mathrm{H}, \mathrm{d}, J=8.0 \mathrm{~Hz}, \mathrm{H}-\mathrm{Ts}), 6.37(1 \mathrm{H}, \mathrm{d}, J=11.3$ Hz, H-6), 5.74 (1H, d, J=11.3 Hz, H-7), $4.91(1 \mathrm{H}, \mathrm{s}$, H-19), 4.88 (1H, s, H-19), 4.48 (1H, m, H-1), 4.45 $(1 \mathrm{H}, \mathrm{m}, \mathrm{H}-3), 3.92(1 \mathrm{H}, \mathrm{m}, \mathrm{H}-22), 3.90(1 \mathrm{H}, \mathrm{m}, \mathrm{H}-$ 22), $2.75(1 \mathrm{H}, \mathrm{m}), 2.43(1 \mathrm{H}, \mathrm{m}), 2.37\left(3 \mathrm{H}, \mathrm{s}, \mathrm{CH}_{3}-\right.$ Ts), $2.17(1 \mathrm{H}, \mathrm{m}), 1.85(3 \mathrm{H}, \mathrm{m}), 1.55(5 \mathrm{H}, \mathrm{m}), 1.37$ $(3 \mathrm{H}, \mathrm{m}), 1.15(3 \mathrm{H}, \mathrm{m}), 0.93(3 \mathrm{H}, \mathrm{d}, J=6.5 \mathrm{~Hz}, \mathrm{H}-21)$, $0.84\left(9 \mathrm{H}, \mathrm{s}, \mathrm{CH}_{3}\right.$-terc-BuSi), $0.80\left(9 \mathrm{H}, \mathrm{s}, \mathrm{CH}_{3}\right.$-terc$\mathrm{BuSi}), 0.44$ (3H, s, H-18), 0.00 (12H, s, $\left.\mathrm{CH}_{3}-\mathrm{SiMe}\right)$; ${ }^{13} \mathrm{C}-\mathrm{NMR}\left(\mathrm{CDCl}_{3}, \delta\right): 154.0$ (C-10), 145.0 (C-8), 142.9 (C-Ts), 136.1 (C-5), 133.6 (C-Ts), 130.2 (CHTs), 128.3 (CH-6), 122.0 (CH-7), $107.1\left(\mathrm{CH}_{2}-19\right)$, $75.9\left(\mathrm{CH}_{2}-22\right), 70.6(\mathrm{CH}-1), 67.2(\mathrm{CH}-3), 56.4(\mathrm{CH}-$ 14), 52.6 (CH-17), 46.2 (C-13), $44.3\left(\mathrm{CH}_{2}\right), 40.6$ $\left(\mathrm{CH}_{2}\right), 37.0(\mathrm{CH}-20), 36.9\left(\mathrm{CH}_{2}\right), 29.2\left(\mathrm{CH}_{2}\right), 27.3$ $\left(\mathrm{CH}_{2}\right), 26.3\left(\mathrm{CH}_{3}\right.$-terc-BuSi), $26.3\left(\mathrm{CH}_{3}\right.$-terc-BuSi), $26.2\left(\mathrm{CH}_{3}\right.$-terc-BuSi), $23.8\left(\mathrm{CH}_{2}\right), 22.6\left(\mathrm{CH}_{2}\right), 22.0$ $\left(\mathrm{CH}_{3}\right.$-Ts), 18.6 (C-terc-BuSi), 18.4 (C-terc-BuSi), $17.4\left(\mathrm{CH}_{3}-18\right), 12.4\left(\mathrm{CH}_{3}-21\right),-4.4\left(\mathrm{CH}_{3}-\mathrm{SiMe}\right),-4.5$ $\left(\mathrm{CH}_{3}-\mathrm{SiMe}\right),-4.5\left(\mathrm{CH}_{3}-\mathrm{SiMe}\right)$;

LRMS: : $[\mathrm{m} / \mathrm{z} \%]: 729.35\left[(\mathrm{M}+1)^{+},(23)\right], 728.35$ $\left[\mathrm{M}^{+},(22)\right], 727.34\left[\mathrm{M}^{+}-1,(14)\right], 597.28(32), 596.28$

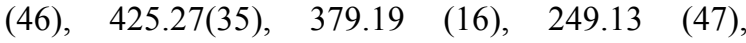
248.13(100), 247.11 (33).

HRMS: $\mathrm{m} / \mathrm{z}$ calcd $\mathrm{C}_{41} \mathrm{O}_{5} \mathrm{Si}_{2} \mathrm{SH}_{68}$ for: 729.4404 ; found: 729.4418

(((1R,3S,E)-5-((E)-2-((3aS,7aR)-1-((S)-1azidopropan-2-yl)-7a-methylhexahydro- $1 H$ inden-4(2H)-ylidene)ethylidene)-4methylenecyclohexane-1,3-diyl)bis(oxy))bis(tertbutyldimethylsilane) (11)

To a solution of tosylate $\mathbf{1 8}(933 \mathrm{mg}, 1.28 \mathrm{mmol})$ in DMF $(15 \mathrm{~mL})$ was added $\mathrm{NaN}_{3}(832 \mathrm{mg}, 12.8$ mol) and the mixture was stirred at room temperature for $42 \mathrm{~h} . \mathrm{CH}_{2} \mathrm{Cl}_{2}(25 \mathrm{~mL})$ was added and the organic phase was washed with $\mathrm{H}_{2} \mathrm{O}(3 \times 15 \mathrm{~mL})$, dried $\left(\mathrm{Na}_{2} \mathrm{SO}_{4}\right)$, filtered and concentrated in vacuo to afford a residue which was chromatographed on silica gel using 5\% EtOAc/Hexane as solvent, affording $750 \mathrm{mg}(97 \%)$ of azide 11, as a white solid, M.p.: $104{ }^{\circ} \mathrm{C}, \mathrm{Rf}=0.75$ (10\% EtOAc/Hexane);

${ }^{1}$ H-NMR $\left(\mathbf{C D C l}_{3}, \boldsymbol{\delta}\right): 6.43(1 \mathrm{H}, \mathrm{d}, \mathrm{J}=11.4 \mathrm{~Hz}, \mathrm{H}-6)$, $5.82(1 \mathrm{H}, \mathrm{d}, \mathrm{J}=11.3 \mathrm{~Hz}, \mathrm{H}-7), 4.95(2 \mathrm{H}, \mathrm{d}, \mathrm{J}=16.2$ Hz, H-19), 4.63 (1H, m, H-22), 4.43 (1H, m, H-22), $3.46(1 \mathrm{H}, \mathrm{dd}, \mathrm{J}=, 11.9$ y $3.1 \mathrm{~Hz}, \mathrm{H}-1), 3.05(1 \mathrm{H}, \mathrm{m}$, $\mathrm{H}-3), 2.81(1 \mathrm{H}, \mathrm{m}) ; 2.77(1 \mathrm{H}, \mathrm{m}) ; 2.56(1 \mathrm{H}, \mathrm{m}) ; 2.12$ $(1 \mathrm{H}, \mathrm{m}) ; 1.92(4 \mathrm{H}, \mathrm{m}), 1.85(4 \mathrm{H}, \mathrm{m}) ; 1.63(2 \mathrm{H}, \mathrm{m})$; $1.53\left(3 \mathrm{H}, \mathrm{s}, \mathrm{CH}_{3}-18\right), 1.21(3 \mathrm{H}, \mathrm{m}) ; 1.05(3 \mathrm{H}, \mathrm{d}$, $\left.\mathrm{J}=6.5 \mathrm{~Hz}, \mathrm{CH}_{3}-21\right), 0.89(9 \mathrm{H}, \mathrm{s}$, tert $-\mathrm{BuSi}), 0.85$ (9H, s, tert-BuSi), 0.05 (12 H, s, $\mathrm{CH}_{3}$-TBS);

${ }^{13} \mathbf{C}-\mathbf{R M N}\left(\mathbf{C D C l}_{3}, \boldsymbol{\delta}\right): 153.6(\mathrm{C}-10), 142.7(\mathrm{C}-8)$, 135,7 (C-5), 121.6 (C-6), 116.2 (C-7), $106.7\left(\mathrm{CH}_{2-}\right.$ 19), 70.2 (C-3), 67.2 (C-22), $58.0\left(\mathrm{CH}_{2}\right), 56.2$
(C-14), $53.6(\mathrm{C}-17), 43.9\left(\mathrm{CH}_{2}\right), 40.3\left(\mathrm{CH}_{2}\right), 37.2$ (C-20), $36.6\left(\mathrm{CH}_{2}\right), 28.9\left(\mathrm{CH}_{2}\right), 27.4\left(\mathrm{CH}_{2}\right), 25.9$ $\left(\mathrm{CH}_{3}\right.$-tert-BuSi), $23.4\left(\mathrm{CH}_{2}\right), \quad 22.3 \quad\left(\mathrm{CH}_{2}\right), \quad 18.2$ (C-tert-BuSi), 17.9 (C-21), 12.1 (C-18), -4.8 y -4.9 $\left(\mathrm{CH}_{3}-\mathrm{TBS}\right)$

MS (m/z (\%)): 600,43 (M+1, 40); $599.44(\mathrm{M}+, 46)$; 570.43 (21); 542.37 (27); 467.34 (73); 440.32 (20); 248.15 (100);

HRMS: Calcd for $\mathrm{C}_{34} \mathrm{H}_{61} \mathrm{~N}_{3} \mathrm{O}_{2} \mathrm{Si}_{2}: 599.4302$, found: 599.4302 .

(1R,3S, E)-5-((E)-2-((3aS,7aR)-1-((S)-1azidopropan-2-yl)-7a-methylhexahydro- $1 H$ inden-4(2H)-ylidene)ethylidene)-4methylenecyclohexane-1,3-diol (19)

To a solution of azide 11 (116 mg, $0.19 \mathrm{mmol})$ in THF (2 mL) was added TBAF $(1.16 \mathrm{~mL}, 1.16 \mathrm{mmol}$, $1 \mathrm{M} \sin$ in THF) and the mixture was stirred for $16 \mathrm{~h}$. Aqueous saturated solution of $\mathrm{NH}_{4} \mathrm{Cl}(10 \mathrm{~mL})$ was added and the product was extracted with EtOAc $(3 \times 10 \mathrm{~mL})$. The combined organic phases were dried $\left(\mathrm{Na}_{2} \mathrm{SO}_{4}\right)$, filtered and concentrated to afford a residue which was chromatographed on silica gel using 50\% EtOAc/Hexane as solvent, affording 72 $\mathrm{mg}(99 \%)$ of azide 19 , as a colourless oil, $\mathrm{Rf}=0.11$ (20\% EtOAc/Hexane);

${ }^{1}$ H-NMR $\left(\mathbf{C D C l}_{3}, \boldsymbol{\delta}\right): 6.55(1 \mathrm{H}, \mathrm{d}, \mathrm{J}=11.5 \mathrm{~Hz}, \mathrm{H}-6)$; $5.86(1 \mathrm{H}, \mathrm{d}, \mathrm{J}=11.5 \mathrm{~Hz}, \mathrm{H}-7) ; 5.10(1 \mathrm{H}, \mathrm{s} ; \mathrm{H}-19)$; $4.95(1 \mathrm{H}, \mathrm{s}, \mathrm{H}-19) ; 4.47\left(1 \mathrm{H}, \mathrm{m}, \mathrm{CH}_{2}-22\right) ; 4.22(1 \mathrm{H}$, $\left.\mathrm{m}, \mathrm{CH}_{2}-22\right) ; 3.37(1 \mathrm{H}, \mathrm{dd}, \mathrm{J}=11.9$ y $3.1 \mathrm{~Hz}, \mathrm{H}-1)$; $3.06(1 \mathrm{H}, \mathrm{m}, \mathrm{H}-3) ; 2.86(1 \mathrm{H}, \mathrm{m}) ; 2.75(1 \mathrm{H}, \mathrm{m}), 2.66$ $(1 \mathrm{H}, \mathrm{m}) ; 2.43(1 \mathrm{H}, \mathrm{m}) ; 1.85(5 \mathrm{H}, \mathrm{m}) ; 1.66(5 \mathrm{H}, \mathrm{m})$; $1.55(2 \mathrm{H}, \mathrm{m}) ; 1.32(3 \mathrm{H}, \mathrm{m}), 1.05,(3 \mathrm{H}, \mathrm{d}, \mathrm{J}=6.6 \mathrm{~Hz}$, $\left.\mathrm{CH}_{3}-21\right) ; 0.56\left(3 \mathrm{H}, \mathrm{s}, \mathrm{CH}_{3}-18\right)$;

${ }^{13}$ C-NMR (CDCl $\left.3, \delta\right): 151.7$ (C-10); 144.5 (C-8); 133,0 (C-5); 123.2 (C-6); 116.1 (C-7); $106.7\left(\mathrm{CH}_{2^{-}}\right.$ 19); 71.1 (C-3); 67.5 (C-22); $57.9\left(\mathrm{CH}_{2}\right) ; 56.2$ $(\mathrm{C}-14) ; 53.6(\mathrm{C}-17) ; 42.0\left(\mathrm{CH}_{2}\right) ; 40.2\left(\mathrm{CH}_{2}\right) ; 37.2$ $(\mathrm{C}-20) ; 36.4\left(\mathrm{CH}_{2}\right) ; 29.0\left(\mathrm{CH}_{2}\right) ; 27.3\left(\mathrm{CH}_{2}\right) ; 23.4$ $\left(\mathrm{CH}_{2}\right) ; 22.3\left(\mathrm{CH}_{2}\right) ; 17.9(\mathrm{C}-21) ; 12.1(\mathrm{C}-18)$;

MS (m/z (\%)): $371.25\left(\mathrm{M}^{+}, 56\right), 354.24$ (35); 322.22 (23); 307.07 (100), 289.07 (44), 273.08 (20);

HRMS: Calcd for $\mathrm{C}_{22} \mathrm{H}_{33} \mathrm{~N}_{3} \mathrm{O}_{2}: 371.2573$, found: 371.2565 .

General procedure for the click chemistry reaction of azide 19 with alkynes $\mathbf{1 2 , 1 3}$ and 14 to afford compounds 20, 21 and 22.

To a solution of azide 19 (70 $\mathrm{mg}, 0.19 \mathrm{mmol})$ in tert-BuOH $(2 \mathrm{~mL})$ and $\mathrm{H}_{2} \mathrm{O}(1 \mathrm{~mL})$ was added a catalytic amount of $\mathrm{CuSO}_{4} .5 \mathrm{H}_{2} \mathrm{O}$, sodium ascorbate ( $13 v \mathrm{~L}$ of $1 \mathrm{M}$ aqueous $\sin$ ), and the chosen alkyne $(0.20 \mathrm{mmol})$. The mixture was stirred at room temperature for $7 \mathrm{~h} . \mathrm{H}_{2} \mathrm{O}(10 \mathrm{~mL})$ was added and the product was extracted with EtOAc $(3 \times 15 \mathrm{~mL})$. The combined organic phases were dried $\left(\mathrm{Na}_{2} \mathrm{SO}_{4}\right)$, filtered and concentrated to afford a residue which was chromatographed on silica gel using 50\% EtOAc/Hexane as solvent to afford the corresponding triazoles $\mathbf{2 0 ,} 21$ or $\mathbf{2 2}$. 
(1R,3S,E)-5-((E)-2-((3aS,7aR)-1-((S)-1-(4-(2hydroxypropan-2-yl)-1H-1,2,3-triazol-1yl)propan-2-yl)-7a-methylhexahydro- $1 H$-inden4(2H)-ylidene)ethylidene)-4-

methylenecyclohexane-1,3-diol (20)

Yield 73\%, Brownish solid, M.p.: $106{ }^{\circ} \mathrm{C}$, $\mathrm{Rf}=0.31$ (EtOAc);

${ }^{1} \mathbf{H}-N M R\left(\mathbf{C D C l}_{3}, \boldsymbol{\delta}\right): 7.38(1 \mathrm{H}, \mathrm{s}, \mathrm{H}-23) ; 6.55(1 \mathrm{H}$, d, J=11.4 Hz, H-6); 5.89 (1H, d, J=11.4 Hz, H-7); 5.19 (1H, s, H-19); 4.96 (1H, s, H-19); 4.57-4.50 (2H, m, H-22); 4.21 (1H, m, H-1); $4.04(1 \mathrm{H}, \mathrm{m}, \mathrm{H}-$ 3); $2.99(2 \mathrm{H}, \mathrm{m}) ; 2.76(1 \mathrm{H}, \mathrm{m}) ; 2.66(1 \mathrm{H}, \mathrm{m}) ; 2.43$ $(1 \mathrm{H}, \mathrm{m}) ; 1.89(5 \mathrm{H}, \mathrm{m}) ; 1.75(4 \mathrm{H}, \mathrm{m}) ; 1.63(6 \mathrm{H}, \mathrm{s}$, $\left.\mathrm{CH}_{3}-25\right) ; 1.43(2 \mathrm{H}, \mathrm{m}) ; 1.21(2 \mathrm{H}, \mathrm{m}) ; 0.87(3 \mathrm{H}, \mathrm{d}$, $\left.\mathrm{J}=6.5 \mathrm{~Hz}, \mathrm{CH}_{3}-21\right) ; 0.60\left(3 \mathrm{H}, \mathrm{s}, \mathrm{CH}_{3}-18\right)$;

${ }^{13} \mathbf{C}-N M R\left(\mathbf{C D C l}_{3}, \boldsymbol{\delta}\right): 155.4(\mathrm{C}-10) ; 151.6(\mathrm{C}-24)$; 144.2 (C-8); 133.2 (C-5);123.6 (C-23); 123.0 (C-6); 116.3 (C-7);109.7 ( $\left.\mathrm{CH}_{2}-19\right) ; 76.7$ (C-25); 71.1 (C-3); $67.5 \quad(\mathrm{C}-1) ; 56.1 \quad(\mathrm{C}-13) ; \quad 55.9 \quad\left(\mathrm{CH}_{2}-22\right) ; \quad 54.1$ (C-17);42.0 $\left(\mathrm{CH}_{2}\right) ; 40.2\left(\mathrm{CH}_{2}\right) ; 38.2(\mathrm{C}-26$ o $\mathrm{C}-27)$; $36.7\left(\mathrm{CH}_{2}\right), 30.5(\mathrm{C}-26$ o $\mathrm{C}-27) ; 28.9\left(\mathrm{CH}_{2}\right) ; 27.6$ $\left(\mathrm{CH}_{2}\right) ; 25.8(\mathrm{C}-25) ; 23.3\left(\mathrm{CH}_{2}\right) ; 22.4\left(\mathrm{CH}_{2}\right) ; 17.2$ (C-21); 12.2 (C-18);

MS (m/z (\%)): $456.34 \quad(\mathrm{M}+1,40) ; 307.11 \quad(20)$; 289.10 (23); 155.27 (38);

HRMS: Calcd for $\mathrm{C}_{27} \mathrm{H}_{42} \mathrm{~N}_{3} \mathrm{O}_{3}$ : 456.3226, found: 456.3224 .

(1R,3S,E)-5-((E)-2-((3aS,7aR)-1-((S)-1-(4-(3hydroxy-2,4-dimethylpentan-3-yl)-1H-1,2,3triazol-1-yl)propan-2-yl)-7a-methylhexahydro$1 H$-inden-4(2H)-ylidene)ethylidene)-4methylenecyclohexane-1,3-diol (21)

Yield $86 \%$, White solid, M.p.: $123{ }^{\circ} \mathrm{C}, \mathrm{Rf}=0.41$ (EtOAc);

${ }^{\mathbf{1}} \mathbf{H}-\mathbf{N M R}\left(\mathbf{C D C l}_{\mathbf{3}}, \boldsymbol{\delta}\right) \mathbf{0} 7.30(1 \mathrm{H}, \mathrm{s}, \mathrm{H}-23) ; 6.53(1 \mathrm{H}$, d, J=11.3 Hz, H-6); $5.87(1 \mathrm{H}, \mathrm{d}, \mathrm{J}=11.3 \mathrm{~Hz}, \mathrm{H}-7)$; 5.07 (1H, s, H-19); 4.93 (1H, s, H-19); 4.47 (1H. m. $\mathrm{H}-1) ; 4.36(1 \mathrm{H}, \mathrm{m}, \mathrm{H}-3) ; 4.33$ (2H, m, H-22); 2.82 $(2 \mathrm{H}, \mathrm{m}) ; 2,76(2 \mathrm{H}, \mathrm{m}) ; 2.66(2 \mathrm{H}, \mathrm{m}) ; 2.44(2 \mathrm{H}$, $\mathrm{m}) ; 1.66(5 \mathrm{H}, \mathrm{m}) ; 1.43(4 \mathrm{H}, \mathrm{m}) ; 1.22(2 \mathrm{H}, \mathrm{m}) ; 1.11$ $(3 \mathrm{H}, \mathrm{m}), 1.08\left(3 \mathrm{H}, \mathrm{d}, \mathrm{J}=12.8 \mathrm{~Hz}, \mathrm{CH}_{3}-21\right) ; 0.80(12 \mathrm{H}$, $\mathrm{m}, \mathrm{CH}_{3}$-isopropyl); $0.60\left(3 \mathrm{H}, \mathrm{s}, \mathrm{CH}_{3}-18\right)$;

${ }^{13} \mathbf{C}-N M R\left(\mathbf{C D C l}_{3}, \boldsymbol{\delta}\right)$ : 171.7 (C-10); 151.7 (C-8); 141.0 (C-24); 133.3 (C-5); 123.4 (C-23); 122.8 (C-6); 117.2 (C-7); $109.6\left(\mathrm{CH}_{2}-19\right) ; 72.0$ (C-25); 70.0 (C-3);65.7 (C-1); 56.1 (C-13); $55.8\left(\mathrm{CH}_{2}-22\right)$; 54.2 (C-17); $41.9\left(\mathrm{CH}_{2}\right) ; 40.1\left(\mathrm{CH}_{2}\right) ; 38.1 ; 36.6$ $\left(\mathrm{CH}_{2}\right) ; 34.1$ (CH-isopropyl); 30.5; $28.9\left(\mathrm{CH}_{2}\right) ; 27.5$ $\left(\mathrm{CH}_{2}\right) ; 23.3\left(\mathrm{CH}_{2}\right) ; 22.4\left(\mathrm{CH}_{2}\right) ; 17.1(\mathrm{C}-21) ; 14.1$ $\left(\mathrm{CH}_{3}\right.$-isopropyl); 12.2 (C-18);

MS (m/z (\%)): $512.53\left(\mathrm{M}^{+}+1,100\right) ; 511.46\left(\mathrm{M}^{+}, 30\right)$ 394.43 (20); 322.31 (34); 307.16 (20); 154.24 (96);

HRMS: Calcd for $\mathrm{C}_{31} \mathrm{H}_{49} \mathrm{~N}_{3} \mathrm{O}_{3}: 511.3852$, found: 511.3868 .
(1R,3S,E)-5-((E)-2-((3aS,7aR)-1-((S)-1-(4-(3hydroxypentan-3-yl)-1H-1,2,3-triazol-1yl)propan-2-yl)-7a-methylhexahydro- $1 H$-inden4(2H)-ylidene)ethylidene)-4methylenecyclohexane-1,3-diol (22)

Yield $87 \%$, White solid, M.p.: $110^{\circ} \mathrm{C}, \mathrm{Rf}=0.41$ (EtOAc);

${ }^{1} \mathbf{H}-N M R\left(\mathbf{C D C l}_{3}, \boldsymbol{\delta}\right): 7.37(1 \mathrm{H}, \mathrm{s}, \mathrm{H}-23) ; 6.55(1 \mathrm{H}$, $\mathrm{d}, \mathrm{J}=11.4 \mathrm{~Hz}, \mathrm{H}-6) ; 5.89(1 \mathrm{H}, \mathrm{d}, \mathrm{J}=11.4 \mathrm{~Hz}, \mathrm{H}-7)$; 5.09 (1H, s, H-19); 4.96 (1H, s, H-19); 4.47 (1H, m, $\mathrm{H}-1), 4.36(1 \mathrm{H}, \mathrm{m}, \mathrm{H}-3) ; 4.33$ (2H, m, H-22); 2.82 $(2 \mathrm{H}, \mathrm{m}) ; 2.76(2 \mathrm{H}, \mathrm{m}), 2.66(1 \mathrm{H}, \mathrm{m}) ; 2.43(1 \mathrm{H}, \mathrm{m})$; 2.05 (4H,m, $\left.\mathrm{CH}_{2}-\mathrm{Et}\right) ; 1.88(4 \mathrm{H}, \mathrm{m}) ; 1.66(5 \mathrm{H}, \mathrm{m})$; $1.43(3 \mathrm{H}, \mathrm{m}) ; 1.22(3 \mathrm{H}, \mathrm{m}) ; 0.85\left(9 \mathrm{H}, \mathrm{m}, \mathrm{CH}_{3}\right.$-Et y $\left.\mathrm{CH}_{3}-21\right) ; 0.60\left(3 \mathrm{H}, \mathrm{s}, \mathrm{CH}_{3}-18\right)$;

${ }^{13} \mathbf{C}-N M R\left(\mathbf{C D C l}_{3}, \boldsymbol{\delta}\right): 151.7$ (C-10); 144.6 (C-24); 144.6 (C-8); 133.3 (C-5); 123.0 (C-23); 119.6 (C-6); $116.3 \quad(\mathrm{C}-7) ; 109.7 \quad\left(\mathrm{CH}_{2}-19\right) ; \quad 76.7(\mathrm{C}-25) ; \quad 71.1$ (C-3);65.8 (C-1); 56.2 (C-13); $55.9\left(\mathrm{CH}_{2}-22\right) ; 54.2$ (C-17); $42.0\left(\mathrm{CH}_{2}\right) ; 40.2\left(\mathrm{CH}_{2}\right) ; 38.2 ; 36.7\left(\mathrm{CH}_{2}\right)$; $33.9\left(\mathrm{CH}_{2}\right.$-Et); 30.5; $28.9\left(\mathrm{CH}_{2}\right) ; 27.5\left(\mathrm{CH}_{2}\right) ; 23.3$ $\left(\mathrm{CH}_{2}\right) ; 22.4\left(\mathrm{CH}_{2}\right) ; 17.1(\mathrm{C}-21) ; 12.2(\mathrm{C}-18) ; 7.8$ $\left(\mathrm{CH}_{3}-\mathrm{Et}\right)$;

MS (m/z (\%)): $484.51\left(\mathrm{M}^{+}+1,37\right) ; 307.17$ (28); 235.25 (20); 155.26 (33);

HRMS: Calcd for $\mathrm{C}_{29} \mathrm{H}_{46} \mathrm{~N}_{3} \mathrm{O}_{3}: 484.3539$, found: 484.3546 .

Photosensitized isomerization of 20,21 and 22 to afford 15, 16 and 17, using anthracene as triplet sensitizer was carried out following the general procedure described for compound $\mathbf{2 0}$.

To a solution of $20(10 \mathrm{mg}, 0.02 \mathrm{mmol})$ in $\mathrm{MeOH}(10 \mathrm{~mL})$ was added anthracene $(3 \mathrm{mg}, 0.01$ mmol) and a catalytic amount of $\mathrm{Et}_{3} \mathrm{~N}$. The mixture was irradiated with a $200 \mathrm{~W}$ lamp for $6 \mathrm{~h}$, diluted with $\mathrm{CH}_{2} \mathrm{Cl}_{2}(15 \mathrm{~mL})$ and washed with brine $(10$ $\mathrm{mL}$ ). After solvent evaporation the resulting residue was chromatographed on silica gel using $\mathrm{CH}_{2} \mathrm{Cl}_{2}$ and $10 \% \mathrm{MeOH} / \mathrm{CH}_{2} \mathrm{Cl}_{2}$ as solvent, affording $9 \mathrm{mg}$ (90\%) of analogue $\mathbf{1 5}$.

(1R,3S,Z)-5-((E)-2-((3aS,7aR)-1-((S)-1-(4-(2hydroxypropan-2-yl)-1H-1,2,3-triazol-1yl)propan-2-yl)-7a-methylhexahydro- $1 H$-inden4(2H)-ylidene)ethylidene)-4methylenecyclohexane-1,3-diol (15)

Yield 90\%, White solid, M.p.: $110{ }^{\circ} \mathrm{C}, \mathrm{Rf}=0.31$ $\left(10 \% \mathrm{MeOH} / \mathrm{CH}_{2} \mathrm{Cl}_{2}\right)$;

${ }^{1}$ H-NMR $\left(\mathbf{C D C l}_{3}, \boldsymbol{\delta}\right): 7.34$ (1H, s, H-23); $6.35(1 \mathrm{H}$, d, J=11.1 Hz, H-6), $6.02(1 \mathrm{H}, \mathrm{d}, \mathrm{J}=11.2 \mathrm{~Hz}, \mathrm{H}-7)$, 5.32 (1H, s, H-19); 4.99 (1H, s, H-19), 4.57-4.50 $(2 \mathrm{H}, \mathrm{m}, \mathrm{H}-22), 4.21(1 \mathrm{H}, \mathrm{m}, \mathrm{H}-1), 4.04(1 \mathrm{H}, \mathrm{m}, \mathrm{H}-$ 3); $2.99(2 \mathrm{H}, \mathrm{m}) ; 2.76(1 \mathrm{H}, \mathrm{m}), 2.66(1 \mathrm{H}, \mathrm{m}) ; 2.43$ $(1 \mathrm{H}, \mathrm{m}) ; 1.89(5 \mathrm{H}, \mathrm{m}) ; 1.75(4 \mathrm{H}, \mathrm{m}) ; 1.63(6 \mathrm{H}, \mathrm{s}$, $\left.\mathrm{CH}_{3}-25\right) ; 1.43(2 \mathrm{H}, \mathrm{m}) ; 1.21(3 \mathrm{H}, \mathrm{m}) ; 0.86(3 \mathrm{H}, \mathrm{d}$, $\left.\mathrm{J}=6.5 \mathrm{~Hz}, \mathrm{CH}_{3}-21\right) ; 0.58\left(3 \mathrm{H}, \mathrm{s}, \mathrm{CH}_{3}-18\right)$; 
${ }^{13} \mathbf{C}-N M R\left(\mathbf{C D C l}_{3}, \boldsymbol{\delta}\right): 155.4(\mathrm{C}-10), 147.6(\mathrm{C}-24)$, 142.2 (C-8); 133.4 (C-5); 124.6 (C-23), 119.6 (C-6); 117.4 (C-7); $111.8\left(\mathrm{CH}_{2}-19\right) ; 76.7(\mathrm{C}-25) ; 70.8$ (C-3); $67.5 \quad$ (C-1);56.3 (C-13); 55.7 $\left(\mathrm{CH}_{2}-22\right) ; \quad 54.1$ (C-17);42.3 ( $\left(\mathrm{CH}_{2}\right) ; 40.2\left(\mathrm{CH}_{2}\right) ; 38.2(\mathrm{C}-26$ o $\mathrm{C}-27)$; $36.7\left(\mathrm{CH}_{2}\right) ; 30.5(\mathrm{C}-26$ o $\mathrm{C}-27) ; 28.9\left(\mathrm{CH}_{2}\right) ; 27.6$ $\left(\mathrm{CH}_{2}\right) ; 25.8(\mathrm{C}-25) ; 23.3\left(\mathrm{CH}_{2}\right) ; 22.4\left(\mathrm{CH}_{2}\right) ; 17.2$ (C-21); 12.2 (C-18);

MS (m/z (\%)): $456.34(\mathrm{M}+1,40), 307.11$ (20), 289.10 (23); 155.27 (38);

HRMS: Calcd for $\mathrm{C}_{27} \mathrm{H}_{42} \mathrm{~N}_{3} \mathrm{O}_{3}$ : 456.3226, found: 456.3224 .

(1R,3S,Z)-5-((E)-2-((3aS,7aR)-1-((S)-1-(4-(3hydroxy-2,4-dimethylpentan-3-yl)-1H-1,2,3triazol-1-yl)propan-2-yl)-7a-methylhexahydro$1 H$-inden-4(2H)-ylidene)ethylidene)-4methylenecyclohexane-1,3-diol (16)

Yield 96\%, colourless oil, $\mathrm{Rf}=0.41(10 \%$ $\mathrm{MeOH} / \mathrm{CH}_{2} \mathrm{Cl}_{2}$ );

${ }^{1} \mathbf{H}-N M R\left(\mathbf{C D C l}_{3}, \boldsymbol{\delta}\right): 7.28(1 \mathrm{H}, \mathrm{s}, \mathrm{H}-23) ; 6.36(1 \mathrm{H}$, d, J=11.4 Hz, H-6); 6.03 (1H, d, J=11.4 Hz, H-7); 5.33 (1H, s, H-19); 4.99 (1H, s, H-19); $4.39(1 \mathrm{H}, \mathrm{m}$, $\mathrm{H}-1) ; 4.36$ (1H, m, H-3); 4.33 (2H, m, H-22); 2.82 $(2 \mathrm{H}, \mathrm{m}), 2,76(2 \mathrm{H}, \mathrm{m}) ; 2.66(2 \mathrm{H}, \mathrm{m}) ; 2.44(2 \mathrm{H}$, $\mathrm{m}) ; 1.66(5 \mathrm{H}, \mathrm{m}) ; 1.43(4 \mathrm{H}, \mathrm{m}) ; 1.22(2 \mathrm{H}, \mathrm{m}) ; 1.11$ $(3 \mathrm{H}, \mathrm{m}), 1.08\left(3 \mathrm{H}, \mathrm{d}, \mathrm{J}=12.8 \mathrm{~Hz}, \mathrm{CH}_{3}-21\right) ; 0.80$ (12H, m, $\mathrm{CH}_{3}$-isopropyl); 0.59 (3H, s, $\left.\mathrm{CH}_{3}-18\right)$;

${ }^{13} \mathrm{C}-\mathrm{NMR}\left(\mathrm{CDCl}_{3}, \mathbf{\delta}\right): 150.7$ (C-10); 147.7 (C-24); 142.3 (C-8); 133.5 (C-5); 124.3 (C-23); 121.4 (C-6); 117.4 (C-7); $111.6 \quad\left(\mathrm{CH}_{2}-19\right) ; 72.0 \quad(\mathrm{C}-25) ; 70.7$ (C-3); 65.7 (C-1); 56.1 (C-13); $55.8\left(\mathrm{CH}_{2}-22\right) ; 54.2$ $(\mathrm{C}-17) ; 41.9\left(\mathrm{CH}_{2}\right) ; 40.1\left(\mathrm{CH}_{2}\right) ; 38.2 ; 36.6\left(\mathrm{CH}_{2}\right)$; 34.1 (CH-isopropyl), 30.48; $28.9\left(\mathrm{CH}_{2}\right) ; 27.5\left(\mathrm{CH}_{2}\right)$; $23.4\left(\mathrm{CH}_{2}\right) ; 22.4\left(\mathrm{CH}_{2}\right) ; 17.1(\mathrm{C}-21) ; 14.1\left(\mathrm{CH}_{3}-\right.$ isopropyl); 12.2 (C-18);

MS (m/z (\%)): 512.53 (100); 394.43 (20); 322.31 (34); 307.16 (20); 154.24 (96);

HRMS: Calcd for $\mathrm{C}_{31} \mathrm{H}_{50} \mathrm{~N}_{3} \mathrm{O}_{2}$ : 512.3852, found: 512.3868

(1R,3S,Z)-5-((E)-2-((3aS,7aR)-1-((S)-1-(4-(3hydroxypentan-3-yl)-1H-1,2,3-triazol-1yl)propan-2-yl)-7a-methylhexahydro- $1 H$-inden4(2H)-ylidene)ethylidene)-4-

methylenecyclohexane-1,3-diol (17)

Yield 90\%, colourless oil, Rf $=0.41(10 \%$ $\mathrm{MeOH} / \mathrm{CH}_{2} \mathrm{Cl}_{2}$ );

${ }^{1} \mathbf{H}-N M R\left(\mathbf{C D C l}_{3}, \boldsymbol{\delta}\right): 7.33(1 \mathrm{H}, \mathrm{s}, \mathrm{H}-23) ; 6.36(1 \mathrm{H}$, d, J=11.4 Hz, H-6), $6.02(1 \mathrm{H}, \mathrm{d}, \mathrm{J}=11.4 \mathrm{~Hz}, \mathrm{H}-7)$; 5.32 (1H, s, H-19); 4.99 (1H, s, H-19); 4.47 (1H. m. $\mathrm{H}-1), 4.36$ (1H, m, H-3); 4.33 (2H, m, H-22);2.82 $(2 \mathrm{H}, \mathrm{m}) ; 2.76(2 \mathrm{H}, \mathrm{m}), 2.66(1 \mathrm{H}, \mathrm{m}) ; 2.43(1 \mathrm{H}, \mathrm{m})$; $2.05\left(4 \mathrm{H}, \mathrm{m}, \mathrm{CH}_{2}-\mathrm{Et}\right) ; 1.88(4 \mathrm{H}, \mathrm{m}) ; 1.66(5 \mathrm{H}, \mathrm{m})$, $1.43(3 \mathrm{H}, \mathrm{m}) ; 1.22(3 \mathrm{H}, \mathrm{m}) ; 0.85\left(9 \mathrm{H}, \mathrm{m}, \mathrm{CH}_{3}-\mathrm{Et} \mathrm{y}\right.$ $\left.\mathrm{CH}_{3}-21\right) ; 0.59\left(3 \mathrm{H}, \mathrm{s}, \mathrm{CH}_{3}-18\right)$;

${ }^{13} \mathbf{C}-N M R\left(\mathrm{CDCl}_{\mathbf{3}}, \boldsymbol{\delta}\right): 151.7$ (C-10); 147.6 (C-24); 142.6 (C-8); 133.4 (C-5); 124.0 (C-23); 120.6 (C-6); $117.3 \quad(\mathrm{C}-7) ; 111.7 \quad\left(\mathrm{CH}_{2}-19\right) ; \quad 76.7(\mathrm{C}-25) ; 71.1$ (C-3);65.8 (C-1); 56.3 (C-13); $56.2\left(\mathrm{CH}_{2}-22\right) ; 54.2$
$(\mathrm{C}-17) ; 42.0\left(\mathrm{CH}_{2}\right) ; 40.2\left(\mathrm{CH}_{2}\right), 38.2 ; 36.7\left(\mathrm{CH}_{2}\right)$; $33.9\left(\mathrm{CH}_{2}-\mathrm{Et}\right) ; 31.5 ; 28.9\left(\mathrm{CH}_{2}\right) ; 27.6\left(\mathrm{CH}_{2}\right) ; 23.2$ $\left(\mathrm{CH}_{2}\right) ; 22.3\left(\mathrm{CH}_{2}\right) ; 17.2(\mathrm{C}-21) ; 12.2(\mathrm{C}-18) ; 7.8$ $\left(\mathrm{CH}_{3}-\mathrm{Et}\right)$;

MS (m/z (\%)): $484.51\left(\mathrm{M}^{+}+1,37\right) ; 307.17$ (28); 235.25 (20); 155.26 (33);

HRMS: Calcd for $\mathrm{C}_{29} \mathrm{H}_{46} \mathrm{~N}_{3} \mathrm{O}_{3}: 484.3539$, found: 484.3546 .

\section{References}

1 - A. W. Norman, Vitamin $D$ the Calcium Homeostatic Steroid Hormone; Academic Press: New York, 1979.

2 - (a) A. W. Norman, R. Bouillon, M. Thomasset, Vitamin D: Chemistry, Biology and clinical Application of the Steroid Hormone; Eds., Vitamin D Workshop: Riverside, CA, 1997; (b) D. Feldman, F. H. Glorieux, J. W. Pike, Vitamin D; Academic Press: San Diego, CA, 1997; (c) R. Pardo, M. Santelli, Synthesis of vitamin D metabolites. Bull. Soc. Chim. Fr. 1985, 98-114; H. Dai, G. H. Posner, Synthetic approaches to vitamin D. Synthesis 1994, 12, 1383-1398; (d) G.-D. Zhu, W. H. Okamura, Synthesis of Vitamin D (Calciferol). Chem. Rev. 1995, 95, 1877-1952; (e) G. H. Posner, M. Kahraman, Organic chemistry of vitamin D analogues (deltanoids). Eur. J. Org. Chem. 2003, 20, 3889-3895.

3 - (a) Y. Fall, M. Torneiro, L. Castedo, A. Mouriño, Tetrahedron Lett.1992, 33, 6683-6686; (b) Y. Fall, M. Torneiro, L. Castedo, A. Mouriño, Tetrahedron, 1997, 53, 4703-4714; (c) Y. Fall, Tetrahedron Lett. 1997, 38, 4909-4912; (d) M. Torneiro, Y. Fall, L. Castedo, A. Mouriño, J. Org. Chem. 1997, 62, 6344-6352; (e) M. De los Angeles Rey, J. A. Martínez-Pérez, A. Fernández-Gacio, K. Halkes, Y. Fall, J. Granja, A. Mouriño, J. Org. Chem. 1999, 64, 31963206; (f) Y. Fall, C. Fernández, C. Vitale, A. Mouriño, Tetrahedron Lett. 2000, 41, 73237326; (g) Y. Fall, C. Fernández, V. González, A. Mouriño, Tetrahedron Lett. 2001, 42, 78157817; (h) Y. Fall, C. Fernández, V. González, A. Mouriño, Synlett. 2001, 10, 1567-1568; (i) Y. Fall, V. González, B. Vidal, A. Mouriño, Tetrahedron Lett. 2002, 43, 427-429; (j) Y. Fall, C. Barreiro, C. Fernández, A. Mouriño, Tetrahedron Lett. 2002, 43, 1433-1436; (k) Y. Fall, O. Diouf, G. Gómez, T. Bolaño, Tetrahedron Lett. 2003, 44, 6069-6072; (1) Suárez, P. L.; Z. Gándara, G. Gómez, Y. Fall, Tetrahedron Lett. 2004, 45, 4619-4621; (m) C. Fernández, O. Diouf, E. Momán, G. Gómez, Y. Fall, Synthesis 2005, 10, 1701-1705; (n) C. Fernández, G. Gómez, C. Lago, E. Momán, Y. Fall, Synlett. 2005, 14, 2163-2166; (o) C. Fernández, Z. Gándara, G. Gómez, B. Covelo, Y. Fall, TetrahedronLett. 2007, 48, 2939-2942; (p) Z. Gándara, M. Pérez, X. Pérez-García, G. 
Gómez, Y. Fall, TetrahedronLett. 2009, 50, 4874-4877; (q) D. G. Salomón, S. M. Grioli, M. Buschiazzo, E. Mascaró, C. Vitale, G. Radivoy, M. Pérez, Y. Fall, E. A. Mesri, A. C. Curino, M. M. Facchinetti, ACS Med. Chem. Lett., 2011, 2, 503-508; (r) M. L. Rivadulla, X. PérezGarcía, M. Pérez, G. Gómez, Y. Fall, TetrahedronLett. 2013, 54, 3164-3166; (s) A. Martínez, Z. Gándara, M. González, G. Gómez, Y. Fall, Tetrahedron Lett. 2013, 54, 3514-3517; (t) M. J. Ferronato, D. G. Salomón, M. E. Fermento, N. A. Gandini, A. L. Romero, M. L. Rivadulla, X. Pérez-García, G. Gómez, M. Pérez, Y. Fall, M. M. Facchinetti, A. C. Curino, Arch. Pharm. Chem. Life Sci. 2015, 348, 315-329.

4 - (a) D. R. Andrews, D. H. R. Barton, R. H. Hesse, M. M. Pechet, J. Org. Chem. 1986, 51, 48194828 and references there cited; (b) M. J. Calverley, Tetrahedron 1987, 43, 4609-4619; (c) S. C. Choudhry, P. S. Belica, D. L. Coffen, A. Focella, H. Maehr, P. S. Manchand, L. Serico, R. T. Yang, J. Org. Chem. 1993, 58, 1496-1500; (d) I. Hijikuro, T. Doi, T. Takahashi, J. Am. Chem. Soc. 2001, 123, 37163722; (e) T. P. Sabroe, H. Pedersen, E. Binderup, Org. Process Res. Dev. 2004, 8, 133-135; (f) H. Shimizu, K. Shimizu, N. Kubodera, K. Yakushijin, D. A. Horne, Tetrahedron Lett. 2004, 45, 1347-1350;

(g) R. M. Moriarty, D. Albinescu, J. Org. Chem. 2005, 70, 7624-7628; (h) W-M. Xu, J. He, M-Q. Yu, G-X. Shen, Org. Lett. 2010, 12, 4431-4433; (i) C. Liu, G-D. Zhao, X. Mao, T. Suenaga, T. Fujishima, C-M. Zhang, Z-P. Liu, Eur. J. Med. Chem. 2014, 85, 569-575; (j) K. Sokolowska, R. R. Sicinski, Steroids, 2014, 87, 67-75; (k) U. Banerjee, A. M. DeBerardinis, M. K. Hadden, Bioorg. Med. Chem. 2015, 23, 548555.

5 - (a) H. C. Kolb, M. G. Finn, K. B. Sharpless, Angew.Chem., Int. Ed. 2001, 40, 2004-2021; (b) V. V. Rostovtsev, L. G. Green, V. V. Fokin, K. B. Sharpless, Angew.Chem., Int. Ed. 2002, 41, 2596-2599; (c) C. W. Toroe, M. Christensen, M. Meldal, J. Org. Chem. 2002, 67, 3057-3064; (d) A. Krasiñski, V. V. Forkin, K. B. Sharpless, Org. Lett.2004, 6, 1237-1240; (e) T. Thirumurugan, D. Matosiuk, K. Jozwiak, Chem. Rev. 2013, 113, 4905-4979.

6 - F. Himo, T. Lowell, R. Hilgraf, V. V. Rostovtsev, L. Noodleman, K. B. Sharpless, V. V. Fokin, J.Am.Chem.Soc. 2005, 127, 210-216. 